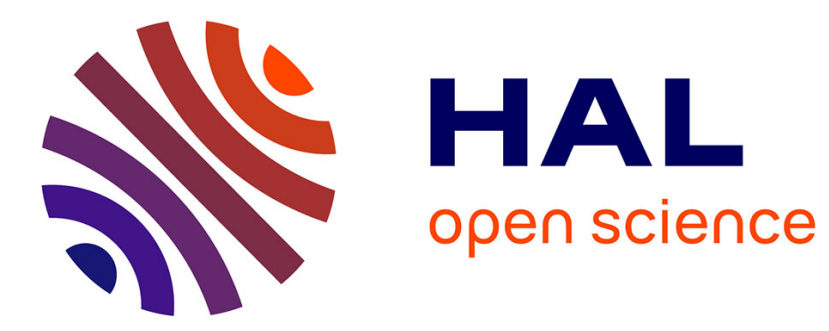

\title{
Crystallography of Complex Forms: The Case of Octocoral Sclerites
}

Daniel Vielzeuf, Nicole Floquet, Jonathan Perrin, Eric Tambutté, Angèle Ricolleau

\section{- To cite this version:}

Daniel Vielzeuf, Nicole Floquet, Jonathan Perrin, Eric Tambutté, Angèle Ricolleau. Crystallography of Complex Forms: The Case of Octocoral Sclerites. Crystal Growth \& Design, 2017, 17 (10), pp.5080 - 5097. 10.1021/acs.cgd.7b00087 . hal-01720343

\section{HAL Id: hal-01720343 https://hal.science/hal-01720343}

Submitted on 30 Apr 2018

HAL is a multi-disciplinary open access archive for the deposit and dissemination of scientific research documents, whether they are published or not. The documents may come from teaching and research institutions in France or abroad, or from public or private research centers.
L'archive ouverte pluridisciplinaire HAL, est destinée au dépôt et à la diffusion de documents scientifiques de niveau recherche, publiés ou non, émanant des établissements d'enseignement et de recherche français ou étrangers, des laboratoires publics ou privés. 


\section{The Crystallography of complex forms: the case of octocoral sclerites}

Daniel Vielzeuf, ${ }^{* a}$ Nicole Floquet, ${ }^{a}$ Jonathan Perrin, ${ }^{a}$ Eric Tambutté, ${ }^{b}$ and Angèle Ricolleau ${ }^{a}$

${ }^{a}$ Aix Marseille Univ, CNRS, CINAM, Marseille, France, Europe

${ }^{b}$ Centre Scientifique de Monaco, Department of marine biology, MC-98000, Monaco

* Corresponding author: D. Vielzeuf

Address: Aix Marseille Univ, CNRS, CINAM UMR 7325, 13288 Marseille, France, Europe

E-mail addresses: vielzeuf@ cinam.univ-mrs.fr (D.Vielzeuf), floquet@ cinam.univ-mrs.fr (N.

Floquet), perrin@cinam.univ-mrs.fr (J. Perrin), etambutte@centrescientifique.mc (E. Tambutté), ricolleau@ cinam.univ-mrs.fr (A. Ricolleau)

Abstract : Octocorals sclerites are ideal structures to study the emergence of complex shapes from particular arrangements of crystallites in biominerals. Sinularia polydactyla sclerites have been studied by polarizing microscopy, scanning electron microscopy, and electron backscattered diffraction. Small sclerites $(<100 \mu \mathrm{m})$ are simple mesocrystalline structures, with similarly oriented submicrometer crystallites arranged in elongated fibers, with only a low degree of ordered misorientations between them. Large sclerites $(\sim 2 \mathrm{~mm})$ are composite structures: (1) at their center, small proto-sclerites $(\leq 20 \mu \mathrm{m})$ are present and act as nuclei around which growth proceeds. (2) The large sclerite axial frame is made of crystallites with c axes arranged in three directions, at an inclination of about $22^{\circ}$ from the long sclerite axis. The axial frame displays a center of symmetry and crystallites are arranged in opposite trihedral arrangement with respect to this center. (3) Inside the large sclerite, deeply rooted tubercles develop with crystallite c axes close to perpendicular to the long sclerite axis. The tubercles grow by a branching process and display crystallite misorientations ordered around the three a axes of the hexagonal unit cell of calcite. Crystallites in both the frame and the tubercles form trigonal inverse pyramids resulting from sudden or progressive changes of crystallite orientations. A crystallographic model emphasizes the importance of the trigonal inverse pyramid as a structural pattern. Concerning the sclerite morphology, small crystallite sizes, ordered misorientations and mesotwinning are important features to achieve concave shapes. The sclerite surface morphology results from the regulation of mesocrystalline growth and patterning by cells or vacuoles. In this respect, the sclerite morphology is the product of internal and external forces, among which crystallographic order and molding play important roles. 


\section{Introduction}

In his seminal article on the chemistry of form ${ }^{1}$, Mann pointed out several facts concerning biominerals: they do not look like the same mineral formed in chemical or geological systems; their complex shapes bear no resemblance to the underlying crystal structure; biominerals depart from their inorganic counterparts as they are ordered but non periodic; curvature is part of the order, and their architecture is constructed hierarchically on length scales from the nanometer to millimeter level. To explain these features, Mann considers that biomineralized structures originate from the "vectorial regulation of crystal growth and patterning in or between organic assemblies such as vesicles and polymeric frame. The elaborate inorganic shapes arise from replication of the associated organic matrix through processes that are analogous to a cast produced in a mould'. In this model, organic patterning rather than intrinsic structural or crystallographic parameters are favored for the formation of complex biomineral forms. In a parallel series of studies, Addadi et al. ${ }^{2}$ and Weiner et al. ${ }^{3}$, considered that 'molding' crystalline materials into shapes adapted to biological functions is difficult since crystalline materials tend to adopt specific shapes reflecting their internal symmetry. To solve this conundrum, these authors proposed that amorphous materials could be alternatives to crystalline materials as they could 'be shaped more easily by the space in which they form'. Since then, the idea that amorphous inorganic materials such as amorphous calcium carbonate (ACC) act as moldable precursors essential to the formation of complex hierarchical assemblages has expanded in the biomineralogy community ${ }^{4}$.

In these works that opened new ways of thinking about biomineralization processes, limited importance has been given to intrinsic processes that allow crystalline materials to acquire any kind of functional shape ${ }^{5}$. The aim of the present article is to address this question using 
octocoral sclerites as models. Sclerites are grains of Mg-calcite found in the living tissues of various organisms, in particular octocorals. Because of their sizes, their crystallographic order, and variable degree of complexity, sclerites are suitable objects to study the transition from simple to complex crystallographic structures. In 1924, Schmidt ${ }^{6}$ determined the calcite crystallographic $c$ axis orientations of several octocoral sclerites and identified four different types summarized by Bengtson ${ }^{7}$ as (1) spindle-shaped sclerites consisting of a single crystal of calcite with $c$ axis perpendicular to the long sclerite axis; (2) spindle- or rod-shaped smooth or tuberculated sclerites consisting of small acicular crystallites with long axes parallel to their $c$ axes and roughly parallel to the long sclerite axis; (3) scale-shaped or rounded sclerites consisting of calcite in spherulitic aggregates with $c$ axes radiating from the center; (4) spindle-shaped tuberculated sclerites with $c$ axis directions roughly parallel to the long sclerite axis, except in the tubercles showing $c$ axes oriented in the direction of the tubercle, i.e. perpendicular to the sclerite surface. So far, this classification has not been challenged, but quantitative determination of crystallographic properties of octocoral sclerites are scarce ${ }^{8}$. Below, using octocoral sclerites of the genus Sinularia Polydactyla as a model, the emergence of complex shapes from particular arrangements of crystalline units during biomineral growth is discussed together with intrinsic parameters that provide the required degrees of freedom for aggregates of calcite crystallites to make all sorts of shapes.

\section{Materials and Methods}

Materials : Sclerites were extracted from a colony of Sinularia Polydactyla (Cnidaria, Anthozoa, Octocorallia), a branching coral grown in aquaria at the Centre Scientifique de Monaco, in controlled culture. Aquaria conditions were semi-open circuit, Mediterranean seawater heated to $25 \pm 0.5^{\circ} \mathrm{C}$, salinity of $38.2 \mathrm{psu}$, illuminated with HQI-10000K; BLV-Nepturion at a constant 
irradiance of $175 \mu \mathrm{mol}$ photons $\mathrm{m}^{-2} \mathrm{~s}^{-1}$ on a $12 \mathrm{~h}$ day-night cycle. Corals were fed three times a week with a mix of Artemia salina nauplii and A. salina frozen adults, and frozen krill.

Polarized light microscopy was performed on a Zeiss Scope A1 microscope equipped in transmission and reflection mode, bright or dark field, with rotating stage and polarizer/analyzer. Five magnifications $(25,50,100,200$ and 500x) are available. Images were collected with a Canon digital camera mounted on the microscope, transferred to a computer and processed to enhance contrast and remove the background. Isolated sclerites were examined in both reflected and transmitted light. Polished sections of sclerites mounted in epoxy were observed by reflection, and polarized light with or without analyzer.

Scanning electron microscopy (secondary electron imaging SEM-SE) was carried out on a JEOL 6320F coupled with a field emission gun. Separate sclerites were dispersed on the sample holder and coated with carbon prior to analysis. Depending on the sample, accelerating voltage and working distances conditions varied within the range $3-15 \mathrm{kV}$ and 6-15 mm, respectively.

Scanning electron microscopy (BackScattered Electron imaging SEM-BSE) was performed on a Raith Pioneer at CINaM, Marseille. For these observations, some Sinularia sclerites were embedded in epoxy, with the long axis either parallel or perpendicular to the plane of observation. The mounts were polished with diamond paste down to $0.1 \mu \mathrm{m}$ and observed using the following conditions: $20 \mathrm{kV}$ accelerating voltage, $9.5 \mathrm{nA}$ probe current and $6 \mathrm{~mm}$ working distance. One of the difficulties in studying hierarchical structures is to bridge the spatial scales. The Raith Pionner SEM used in this study is equipped with a programmable high precision moving stage allowing the completion of large mosaics of high resolution images. Data were automatically acquired during sessions that lasted 8-10 hours. Images were assembled and processed to homogenize contrast and remove side effects using routines developed for the 
purpose $^{9}$. The end-product is an easy-access numeric image covering large sample surface, in focus and at high resolution, bridging the scales from $100 \mathrm{~nm}$ to a few $\mathrm{mm}$.

\section{Electron microprobe (EMP)}

A chemical map of magnesium was obtained in a large sclerite on an SX100 Cameca electron microprobe at Laboratoire Magmas et Volcans, Clermont-Ferrand, France. The following analytical conditions were used for the $650 \times 300$ pixel map: $15 \mathrm{kV}$ acceleration voltage, $40 \mathrm{nA}$ beam current, $1 \mu \mathrm{m}$ electron beam diameter, and step interval of $1 \mu \mathrm{m}$. The sample was coated with a $\sim 20 \mathrm{~nm}$ carbon layer.

Electron BackScattered Diffraction (EBSD) measurements were made on a CamScan X500FE CrystalProbe at Geosciences Montpellier, using an accelerating voltage of $17 \mathrm{kV}$, a probe current of $3.5 \mathrm{nA}$, a working distance of $25 \mathrm{~mm}$ and step intervals from 0.5 to $5 \mu \mathrm{m}$ at a speed of $\sim 0.1 \mathrm{~s}$ per step. EBSD samples were prepared by conventional polishing using diamond paste with grit sizes down to $0.1 \mu \mathrm{m}$, followed by colloidal silica. The CamScan CrystalProbe is equipped with an Oxford/HKL technology "channel 5" EBSD system. The mean angular uncertainties are less than $1^{\circ}$. EBSD patterns were indexed using the crystallographic data of Mg calcite (Space group 167, hexagonal setting, lattice parameters $a=4.941 \AA$ and $c=16.864 \AA$ ). The crystallographic data were analyzed in 3-D space with the 'CaRIne Crystallography' software (http://pagesproorange.fr/carine.crystallography/). In the EBSD maps of crystallographic orientation, calcite planes are color coded using the Euler color key for a hexagonal material. White pixels on the map represent areas where the crystallographic orientation could not be determined. In specified cases, color codes were modified to enhance particular features. Pole figures (upper hemisphere stereographic projection) are used to interpret the crystallite orientations. Each pixel on an EBSD map corresponds to a single analysis, and the corresponding pixel in the stereographic projection 
is shown with the same color. Throughout this article, crystallographic notations refer to the unit cell of calcite in the hexagonal setting.

\section{Results and interpretations}

\subsection{General presentation of Sinularia sclerites}

As in many other octocorals ${ }^{10}$, Sinularia sclerites are observed as unfused, individual structures within cell clusters and mesoglea. The spatial arrangement of sclerites inside the tip of a Sinularia branch is shown in Fig. SI\#1 as supplementary information (SI). Sclerites display a large size range: the smallest sclerites collected after removal of the organic tissues are 100 to $200 \mu \mathrm{m}$ long, 10 to $40 \mu \mathrm{m}$, wide, while the largest are $3 \mathrm{~mm}$ long and $0.5 \mathrm{~mm}$ wide. Smaller sclerites are present in the tissues but are easily lost during the separation process. Results obtained on small, then large sclerites will be presented sequentially. The reason for this partitioning will become obvious as the discussion proceeds.

\subsection{Morphology and crystallography of small sclerites}

Small sclerites display an elongated shape, with a pointed end and regularly spaced pods at the other end, giving an overall dart (or club) shape to the structure (Fig. 1a). The SEM images in Figs. 1d and e indicate that these sclerites are made of narrow ( 200 nm) elongated fibers, themselves constituted of small crystallites (Fig. 1e). The fibers probably correspond to the 'acicular crystals' mentioned by Majoran ${ }^{11}$. The BSE-SEM images (Figs. $1 \mathrm{~b}$ and c) of a polished section show a complex internal structure. In the inner part, along the long sclerite axis, small units $10-20 \mu \mathrm{m}$ long and 2-5 $\mu \mathrm{m}$ wide are observed, their shapes are similar to the sclerite (Fig. 1c, white arrows). 


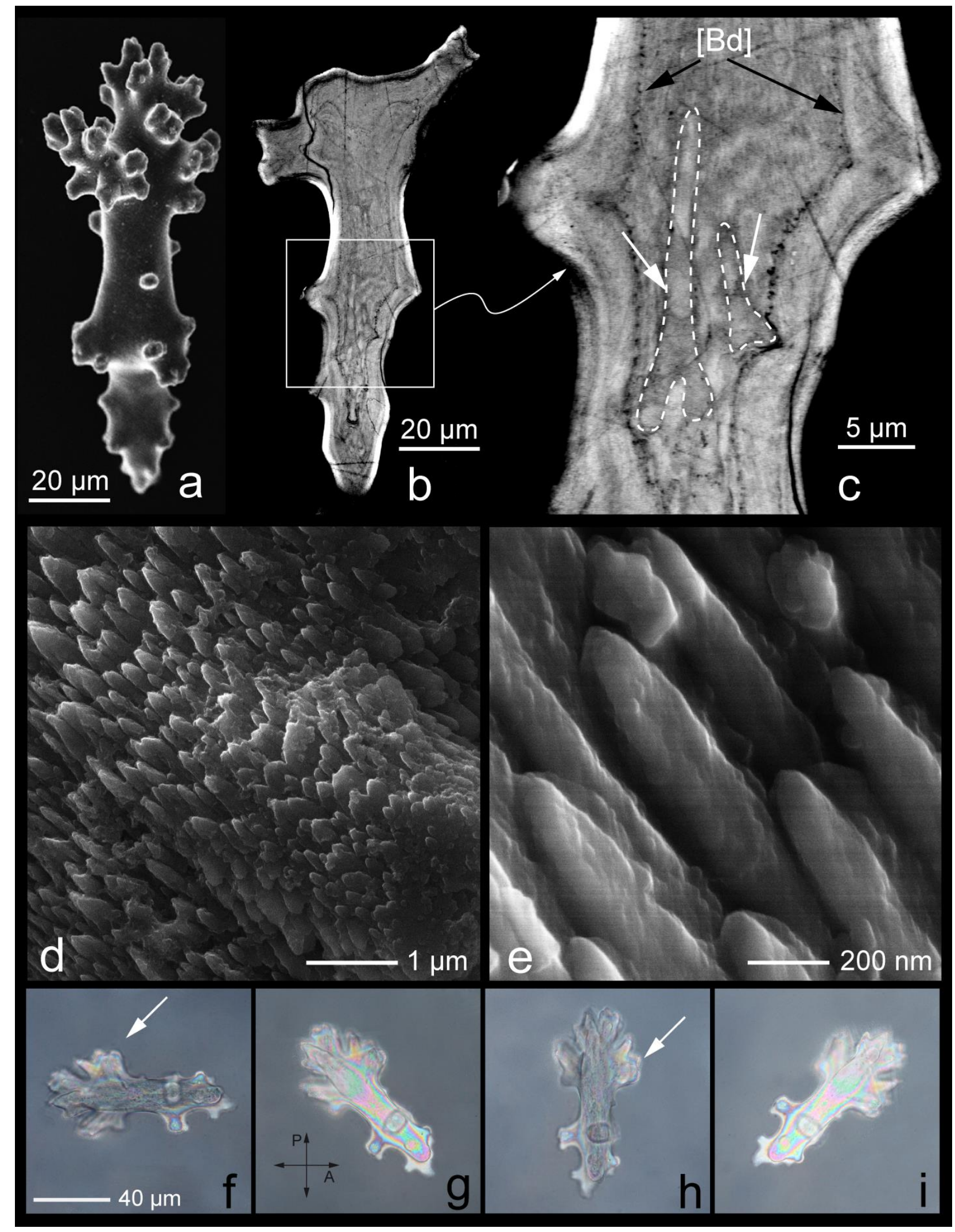


Figure 1: Scanning electron and polarizing microscope images of small Sinularia sclerites. a: SEM-SE image of a small club-shape sclerite. b: SEM-BSE image of a longitudinal polished section of sclerite. $\mathbf{c}$ : Enlargement of $\mathbf{b}$ showing protosclerites inside the small sclerite (white arrows and dotted while lines). The black arrows indicate a boundary between inner and outer sclerite. d and e: SEM-SE images at two magnifications of crystalline fibers made of submicrometer crystalline units. f-i polarizing microscope image of a small sclerite at different rotation angles; transmitted polarized and analyzed light. Note the extinction when the sclerite is parallel or perpendicular to the polarizer or analyzer. Abbreviations: A: analyzer, P: polarizer, Bd: boundary.

These small units are interpreted as sclerites at an early stage of development; they will be referred to as 'proto-sclerites'. In these proto-sclerites, growth features do not look like concentric growth rings; the growth seems to proceed by addition of material at one or both ends of the proto-sclerite (Fig. 1c). The proto-sclerites are embedded in more or less chaotic layers of Mg-calcite, not concentrically arranged around the proto-sclerite (Fig. 1c). Farther away, beyond the boundary labelled [Bd] (Fig. 1c - black arrows), the calcitic layers become concentric and their shapes coincide with the external shape of the sclerite.

In terms of crystallography, polarizing microscopy indicates that small sclerites behave as a single crystal with an overall homogeneous extinction (Fig. 1f-i). This observation implies that the crystallite $c$ axes are roughly parallel to the sclerite long axis. Note that at the tip of lateral microprotuberances (white arrows in Figs $1 \mathrm{f}$ and $\mathrm{h}$ ), the extinction is not simultaneous. It indicates some degree of misorientation among the crystallites, which is consistent with previous reports $^{11}$. A small sclerite has been polished for EBSD analysis (Fig. 2a). The thin white line delineates the sclerite portion that emerged from the epoxy and was analyzed. The EBSD map is shown in Fig. 2b. The blue color corresponds to the most common orientation (indicated by the hexagonal prism), the color change from blue to red indicates a slight and progressive deviation from this orientation. This map and the pole figures of the $\{001\},\{1-10\}$, and $\{104\}$ planes 
(Figs. 2c-e) show that the calcite submicrometer units are similarly oriented, with $c$ axes aligned along the long axis of the sclerite (Fig. 2c). As already stated, the similarity of orientation is not perfect and some dispersion is observed (Figs. 2c to e).
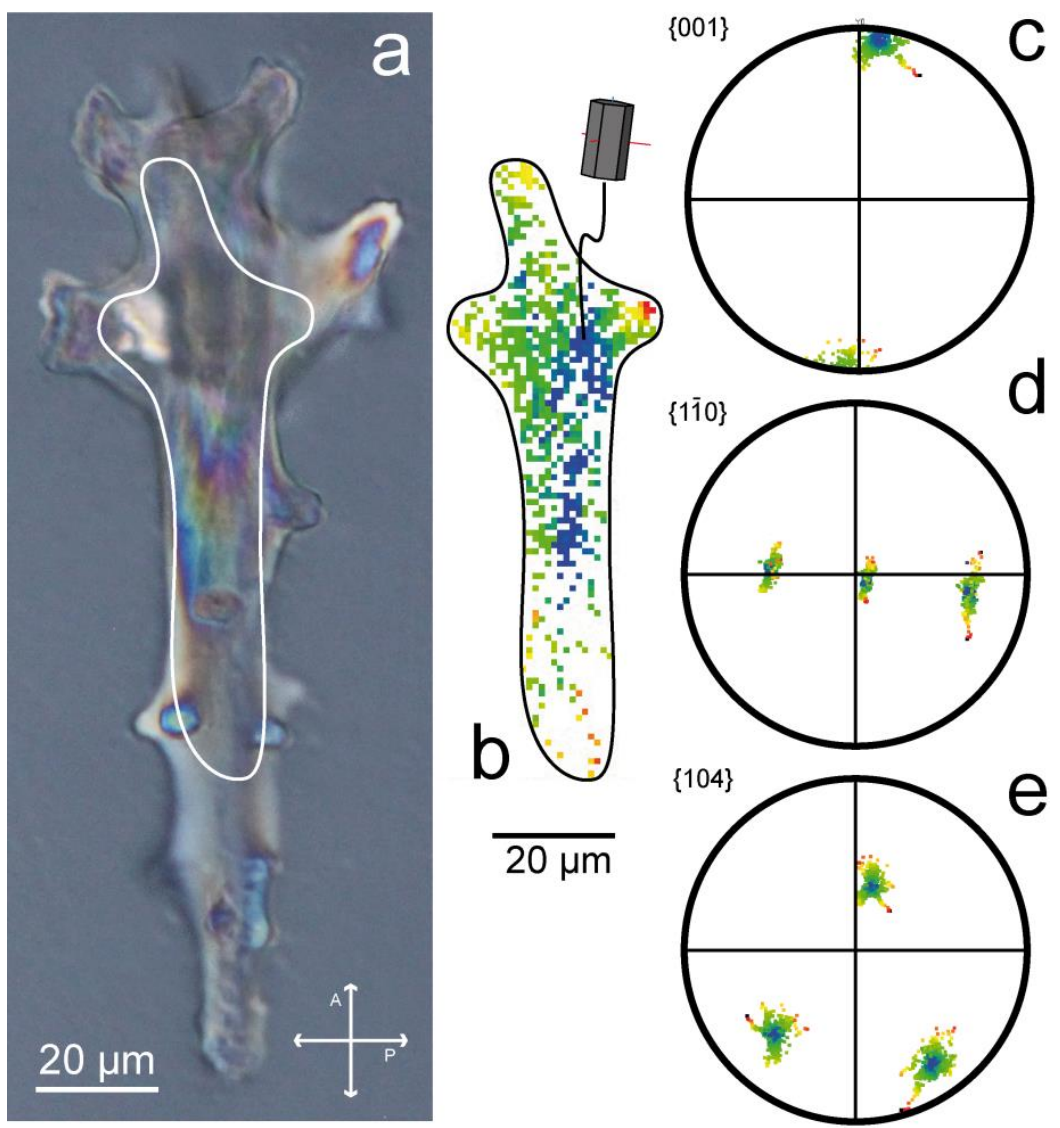

Figure 2: EBSD of a small Sinularia sclerite. a: polarizing microscope image of a small sclerite in transmitted light (crossed polars). The zone contoured with a white line corresponds to the emerged surface studied by EBSD. b: EBSD map of the small sclerite shown in a ( $1 \mu \mathrm{m}$ step, 574 points). The hexagonal prism indicates the main crystallite orientation in the blue area. c: stereographic projections of the $\{001\}\{1-10\}$ and $\{104\}$ planes (upper hemisphere).

If a mesocrystal is defined as a 3D superstructures made of sub-micrometer crystallites in crystallographic register (more details in the discussion), small Sinularia sclerites are relatively simple mesocrystalline structures that can be referred to as 'mono mesocrystalline'. They correspond to type 2 sclerites defined by Schmidt ${ }^{6}$. 


\subsection{Morphology and crystallography of large sclerites}

\subsubsection{Morphology and structural patterns}

Detailed descriptions of large sclerites have been given by Majoran ${ }^{11}$ and Sethmann et al. ${ }^{12}$. Large Sinularia sclerites are spindle-shaped. They are studded with jagged tubercles (Fig. 3a and b). Fractured sclerites without etching reveal a succession of crystalline layers a few micrometer thick (Fig. 4a). The layers are made of columns more or less perpendicular to the surface (Fig. $4 b)$. The lengths of the columns are difficult to estimate but commonly greater than $10 \mu \mathrm{m}$, while their widths are about $\sim 500 \mathrm{~nm}$. Fig. 4b shows that the contrast within a column is not homogeneous which indicates the presence of extremely fine intra-columnar layers $(\sim 100 \mathrm{~nm})$. These fine layers are made of submicrometer units (Fig. 4c). These observations are in agreement with previous descriptions of $\mathrm{HNO}_{3}$-etched cross-sections ${ }^{12}$ showing (1) that the large sclerite inner structure consists of crystalline fibers $\sim 400 \mathrm{~nm}$ thick, in sub-parallel arrangement (Fig. 2e in ${ }^{12}$ ), and (2) that the crystalline fibers are made of 100-200 nm large nanograins (Fig. 2f in ${ }^{12}$ ). Obviously, the columns observed on non-etched sections (Fig. 4b) and the fibers observed by Sethmann et al. ${ }^{12}$ are identical structures observed under different conditions; all the same for the submicrometer units (Fig. 4c) and the nanograins (Fig. $2 \mathrm{f}$ in ${ }^{12}$, see also ${ }^{13}$ ). Interestingly, the fibers shown in the Fig. 2e of Sethmann et al ${ }^{12}$ show a progressive change of orientation that will be discussed further in the EBSD section.

Figures 5 and SI\#2 display two SEM-BSE mosaics showing large sclerite chemical and structural patterns. The alternation of curled layers of different chemical composition is observed. The contrast is mainly due to changes of $\mathrm{Mg} / \mathrm{Ca}$ ratio as demonstrated by the electron microprobe (EMP) map of magnesium displayed in Fig. SI\#3. The composition range determined by EMP is 12.5 to $16.2 \mathrm{~mol} \% \mathrm{MgCO}_{3}$ (mean value: 14.9 , sd: $1.1, \mathrm{n}: 19$ ). 


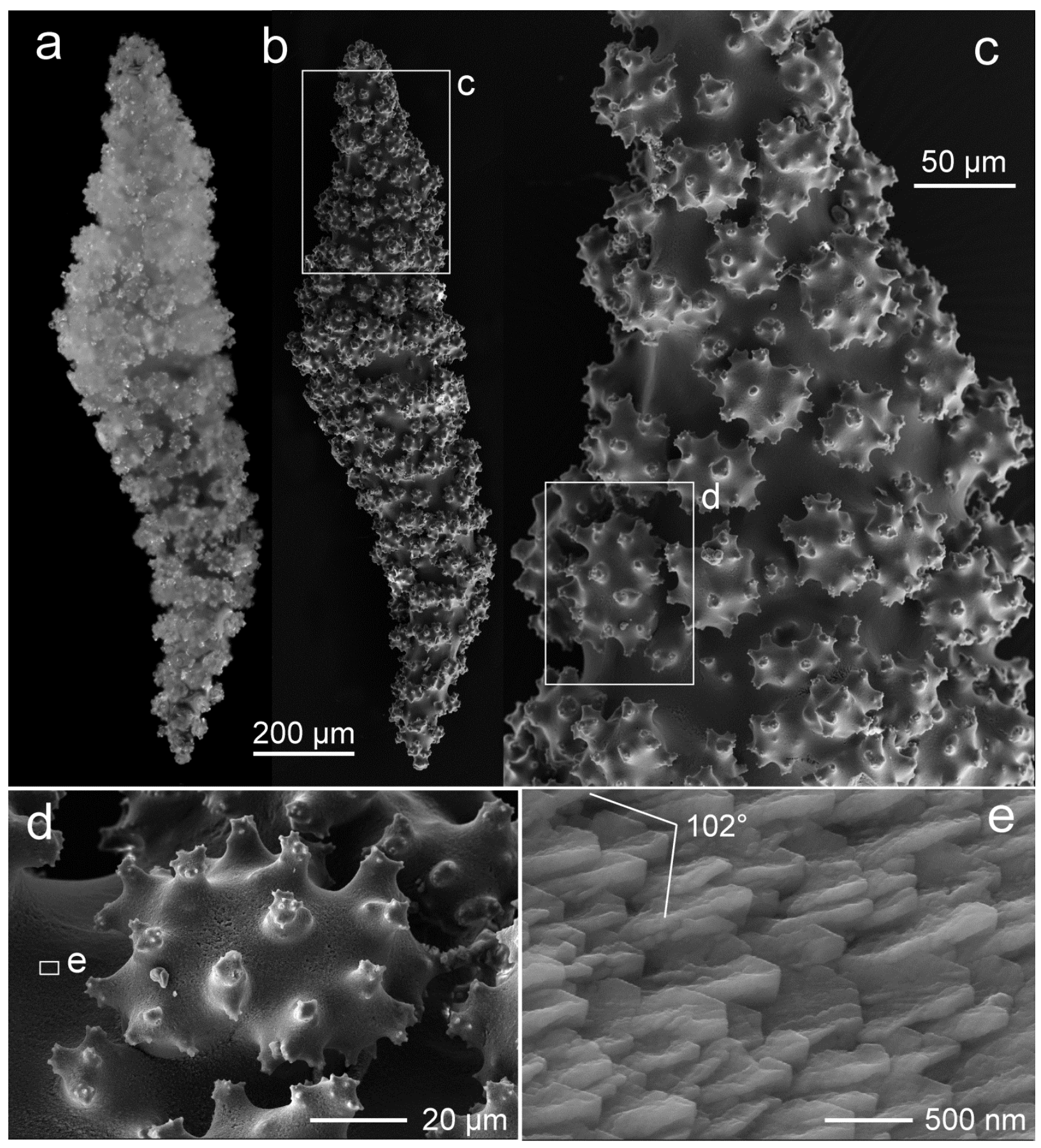

Figure 3: Macroscopic and microscopic features of large Sinularia sclerites. a and b - Stereo microscope and SE-SEM images of a large sclerite. $\mathbf{c}$ - Enlargement of $\mathbf{b}$. d: Detail of a tubercle located in c. e - Crystalline fibers at the surface of the sclerite. Location of the image in $\mathbf{d}$ (rectangle not to scale). Note the angular faces at the growth front. 

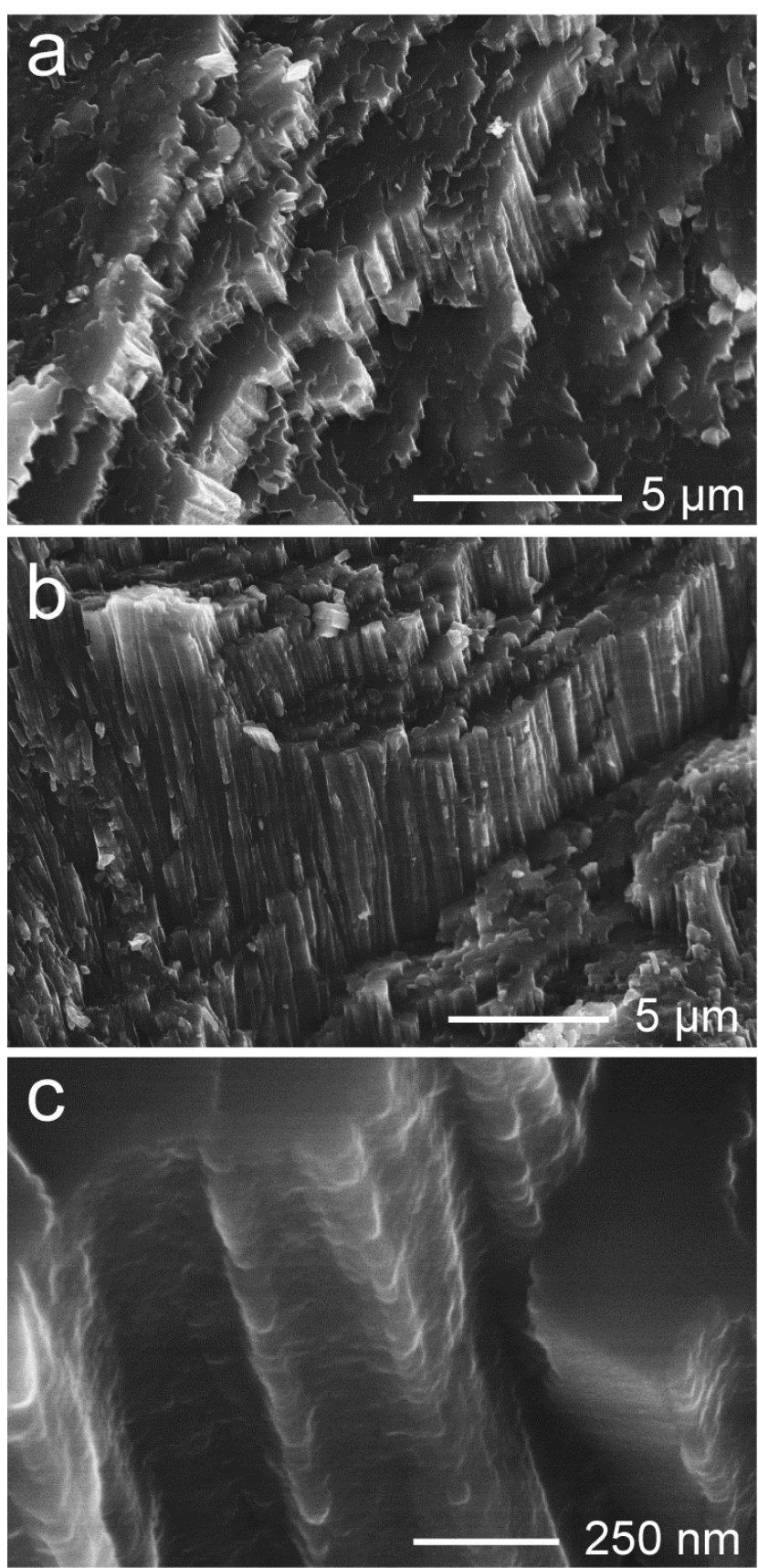

Figure 4: SE-SEM images of a broken section of large Sinularia sclerite. a: Succession of layers made of crystalline columnar fibers. b: Detail of a layer with ultrafine intra layering within the fibers. c: Crystalline fibers made of submicrometer units. 

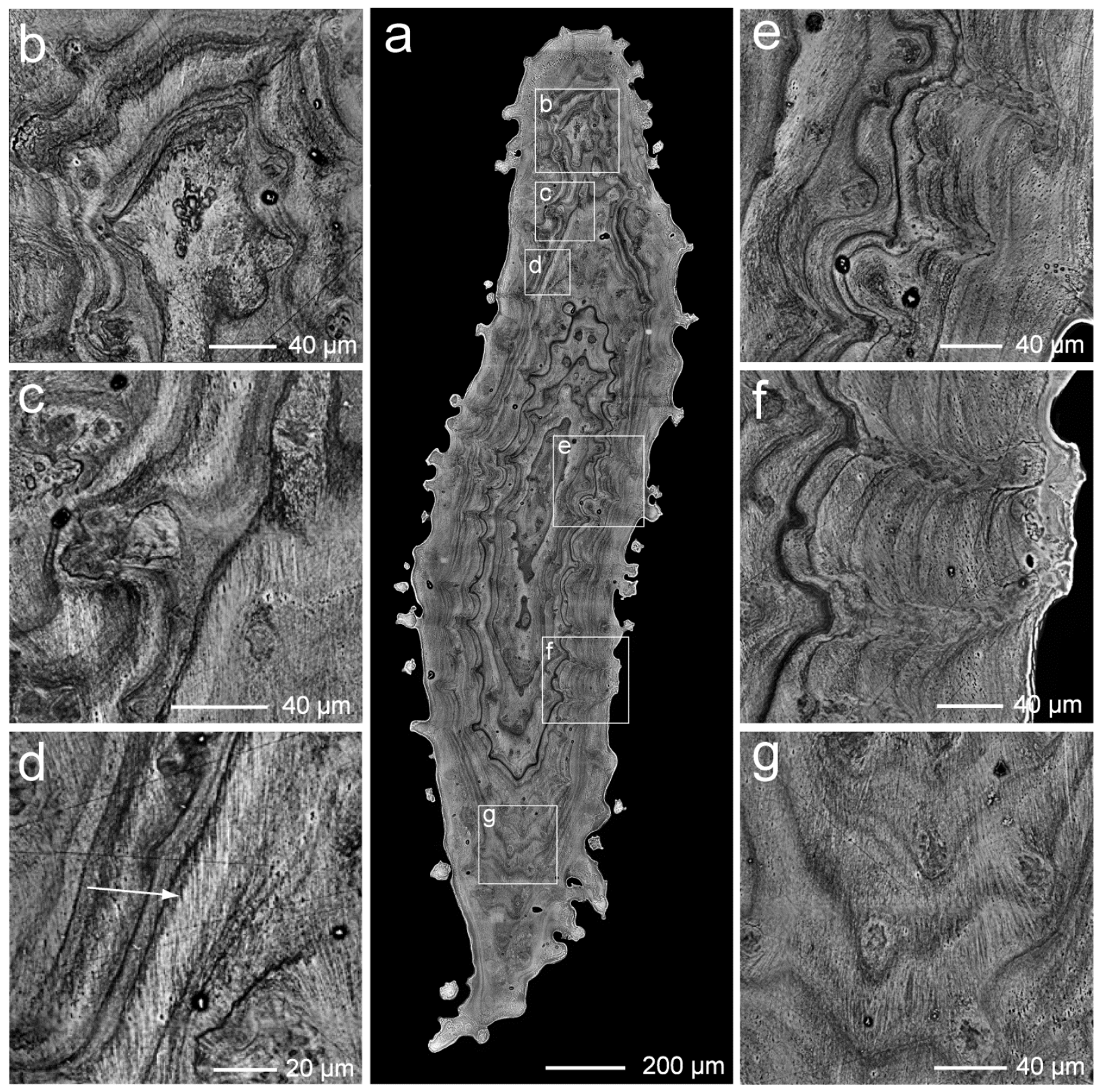

Figure 5: BSE-SEM images of a longitudinal section of a large sclerite. a: mosaic of two hundred and forty three images. $\mathbf{b}$ to $\mathbf{g}$ : enlargement of portions of $\mathbf{a}$. b: protosclerite surrounded by concentric layers. c, $\mathbf{d}$ and $\mathbf{g}$ : crystalline fibers and their angular relationships with the concentric layers, from oblique (c and d) to perpendicular (g). e and f: inner layering in the tubercles. 
This is a surprisingly large compositional range considering that the organism grew in aquarium under constant environmental conditions. The reasons for the presence of chemical zoning under such conditions remain to be determined. The layers are more or less parallel to the external surface and their interfaces represent growth paleosurfaces. The inner part of the large sclerites is often made of dark structures (Fig. SI\#2b to d). Their shape and internal patterns are reminiscent of the proto-sclerites observed in Fig. 1c; thus, these objects are interpreted as proto-sclerites. Note that proto-sclerites are present not only at the center of the sclerite but also along the long inner axis (Figs. 5a and b, SI\#2b to d). The crystalline fibers observed on the SEM-SE images (Fig. 4) can also be seen on the SEM-BSE images (Figs. 5d and g). In some places, crystalline fibers are perpendicular to growth layers (Fig $5 \mathrm{~g}$ ), in agreement with what has been shown in Fig. $4 \mathrm{a}$ and $\mathrm{b}$. But more commonly, the fibers are not perpendicular to growth layers. For instance, in Fig. 5d, the fiber axes and the layer surfaces make an angle of about $28^{\circ}$. Interestingly, interfaces between growth layers are spiky (Fig. 5d - white arrow), as if crystalline fibers displayed crystalline faces at the growth front. The presence of faces is confirmed by the SEM-SE images of the sclerite surface (Fig. 3e). The observation of such faces both inside and at the surface of the sclerite indicates that these features are neither due to late transformations nor preparation artifacts. As already noted by Majoran ${ }^{11}$ and Sethmann et al. ${ }^{12}$, the general fibrous and more or less concentric structure of the sclerite (referred to as the sclerite frame) is disturbed by regularly spaced tubercles (the deeply rooted tubercles) (Figs. 3c and d). For purpose of clarity, the crystallography of the sclerite frame and the deeply rooted tubercles will be discussed one after the other. 


\subsubsection{Crystallography of the large sclerite frame}

\subsubsection{Parallel sections}

The observation of a large sclerite polished section under polarized reflected light without analyzer shows two distinct domains (Fig. 6a): (1) a dark body corresponding to the sclerite frame and (2) brighter and smaller zones elongated perpendicularly to the long sclerite axis. The brighter zones correspond to deeply rooted tubercles. A $45^{\circ}$ stage rotation (Fig. 6b) generates a different pattern with four sectors in opposite arrangement. These sectors are also observed under polarizer and analyzer crossed at an angle $\gamma$ slightly smaller than $90^{\circ}\left(\gamma=84^{\circ}\right)$, (Fig. 6c). For $\gamma$ slightly higher than $90^{\circ}$, the contrast reverses (compare Figs $6 \mathrm{c}$ to $6 \mathrm{e}$, and $6 \mathrm{~d}$ to $6 \mathrm{f}$ ). Under crossed polars at $\gamma=90^{\circ}$, the patterns are not so clear (Fig. 6g and h); however it can be noted that the central axis of the sclerite is extinct in Fig. $6 \mathrm{~g}$. The crystal optics explanation of the features observed in Fig. 6 is provided as Supplementary Information (SI\#4). It emphasizes the interest of observing biominerals under a polarizing microscope with $\gamma$ slightly different than $90^{\circ}$. From the different patterns observed in Fig. 6, qualitative information can be drawn: (1) along the long sclerite axis, crystallites have their $c$ direction close to parallel to that axis, while inside the tubercles, the $c$ axes are perpendicular to the long sclerite axis; (2) two sectors in opposite arrangement with respect to the center of the sclerite have similar arrangements of crystallite orientations.

The EBSD map covering the four sectors observed in Figs 6b, c, e is displayed in Fig. 7b. The exact position of the map in the sclerite is shown in Fig. 7a. In the $\{001\}$ pole figure (Fig. 7c), the near absence of pixels at the center of the projection shows that the calcite crystallites have their $c$ axes approximately in the plane of the map, and that all orientations are present in that plane. This observation may suggest some sort of spherulitic structure; however, the density 
plot of the same data (Fig. 7d) indicates that two orientations prevail. The crystallite $c$ axes lie preferentially on the plane of the map, at an angle of $+/-22^{\circ}$ with respect to the long sclerite axis.
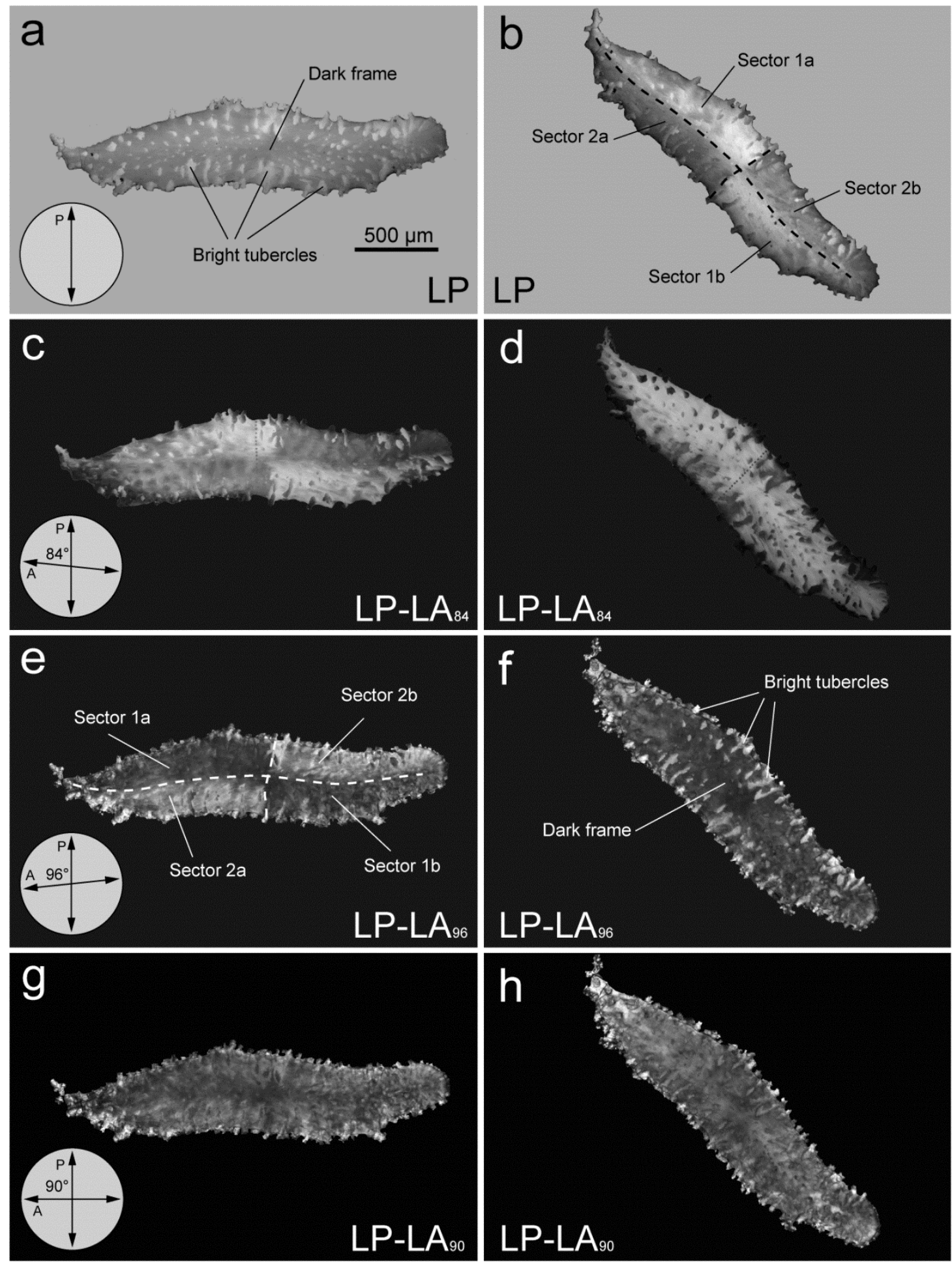
Figure 6: Polarizing microscope images of a longitudinal section of a large sclerite (reflected light). $\mathbf{a}$ and $\mathbf{b}$ : without analyzer ( $\mathbf{b}$ after rotation of $45^{\circ}$ with respect to a). Stage rotations of $90^{\circ}$ generate negative images of $\mathbf{a}$ and $\mathbf{b}$ (not shown here). $\mathbf{c}$ and $\mathbf{d}$ : crossed polars at $84^{\circ}$ ( $\mathbf{d}$ after rotation of $45^{\circ}$ ). Stage rotations of $90^{\circ}$ of $\mathbf{c}$ and $\mathbf{d}$ generate negative images. $\mathbf{e}$ and $\mathbf{f}$ : crossed polars at $96^{\circ}\left(\mathbf{f}\right.$ after rotation of $45^{\circ}$ ). Stage rotations of $90^{\circ}$ of $\mathbf{e}$ and $\mathbf{f}$ generate negative images. $\mathbf{g}$ and h: crossed polars at exactly $90^{\circ}$ (h after rotation of $45^{\circ}$ ). Stage rotations of $90^{\circ}$ of $\mathbf{g}$ and $\mathbf{h}$ generate identical images. See SI\#4 for crystal optics explanation.

These data validate the qualitative observation of sectors under polarizing microscope with crossed polars at $96^{\circ}$ (or $84^{\circ}$ ). Both techniques indicate that large sclerite frames display a sectored organization in $2 \mathrm{D}$, with two preferential directions of crystallite orientations at $+/-22^{\circ}$ with respect to the long sclerite axis. The determination of the $3 \mathrm{D}$ frame structure requires additional observations on transverse sections.

\subsubsection{Transverse sections}

Two transverse sections of large sclerites have been studied by SEM and EBSD. Polarizing microscope and SEM images of transverse section \#1 are displayed in Fig. 8. The polarizing microscope image (Fig. 8a) shows two contrasted domains: a central concentrically layered domain corresponding to the sclerite frame ([F]), and (apparently) unlayered heart-shape zones corresponding to deeply rooted tubercles ([T] in Fig. 8a). The fine polygonal layering in the frame is well marked in the core, and reaches the surface between the heart-shaped structures. Forward- scattered detector (FSD) SEM images (obtained on the CamScan CrystalProbe) enhance topographic contrast and underline the layering with a polygonal shape (Fig. 8c - white arrows). Enlargement of the central part of the sclerite (Fig. 8d) shows that the layer boundaries are spiky indicating that crystalline fibers are facetted at the growth front, as already observed earlier in Figs. 3e and 5d. 

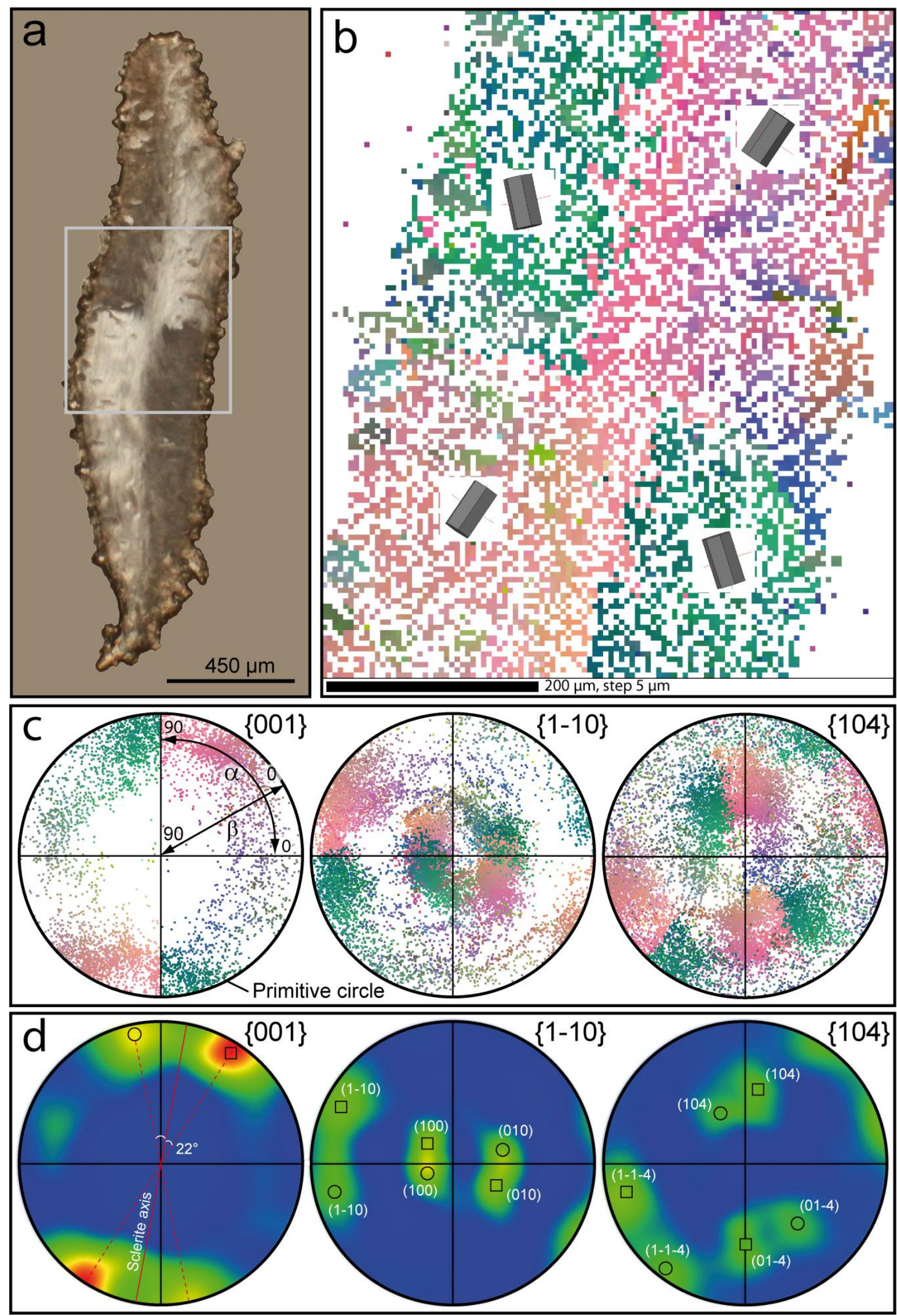
Figure 7: EBSD study of a longitudinal section of a large sclerite. a: location of the map in the sclerite (see also Figs. 5 and 6). b: EBSD map, grid $108 \times 125$, step $5 \mu \mathrm{m}$. c: stereographic projections of the $\{001\},\{1-10\}$, and $\{104\}$ family planes (upper hemisphere). d: Density plot of the stereographic projections shown in c. Circles and squares identify the two main crystallite orientations on the map.

The SEM BSE image (Fig. 8b) shows oscillatory zoning associated with the layering. EBSD data collected at relatively low spatial resolution ( $3 \mu \mathrm{m}$ step interval) on this transverse section are displayed in Fig. 9. The pole figure of the $\{001\}$ planes (Fig. 9c) shows that the calcite crystallites have their $c$ axes scattered in almost all directions, but here again the density plot (Fig. 9d) indicates that three orientations prevail in the frame. These orientations are labelled in red $(R)$, green $(G)$ and blue $(B)$ in Figs $9 b$ and c. Thus, in the frame transverse section, the crystallite $c$ axes are close to perpendicular to the plane of observation, at an angle of $\sim 22^{\circ}$ with respect to the long sclerite axis. Typical crystallite orientations in the three sectors are shown in Fig. $9 \mathrm{~b}$ as three hexagonal prisms. A model of crystallite organization in the sclerite frame will be proposed later in the Discussion.

\subsubsection{Crystallography of deeply rooted tubercles}

Figure 10a is another transverse section of a large Sinularia sclerite under polarizing microscope; two heart-shape zones have been contoured with white dashed lines. These heart shapes display different contrast than the frame and emerge at the surface as tubercles (see Figs. $3 \mathrm{c}$ and $\mathrm{d}$ ). These observations confirm that the heart-shape structures correspond to sections of deeply rooted tubercles. Differences between the frame and the rooted tubercles are observed on SEM FSD images (Figs. 10c and d). 

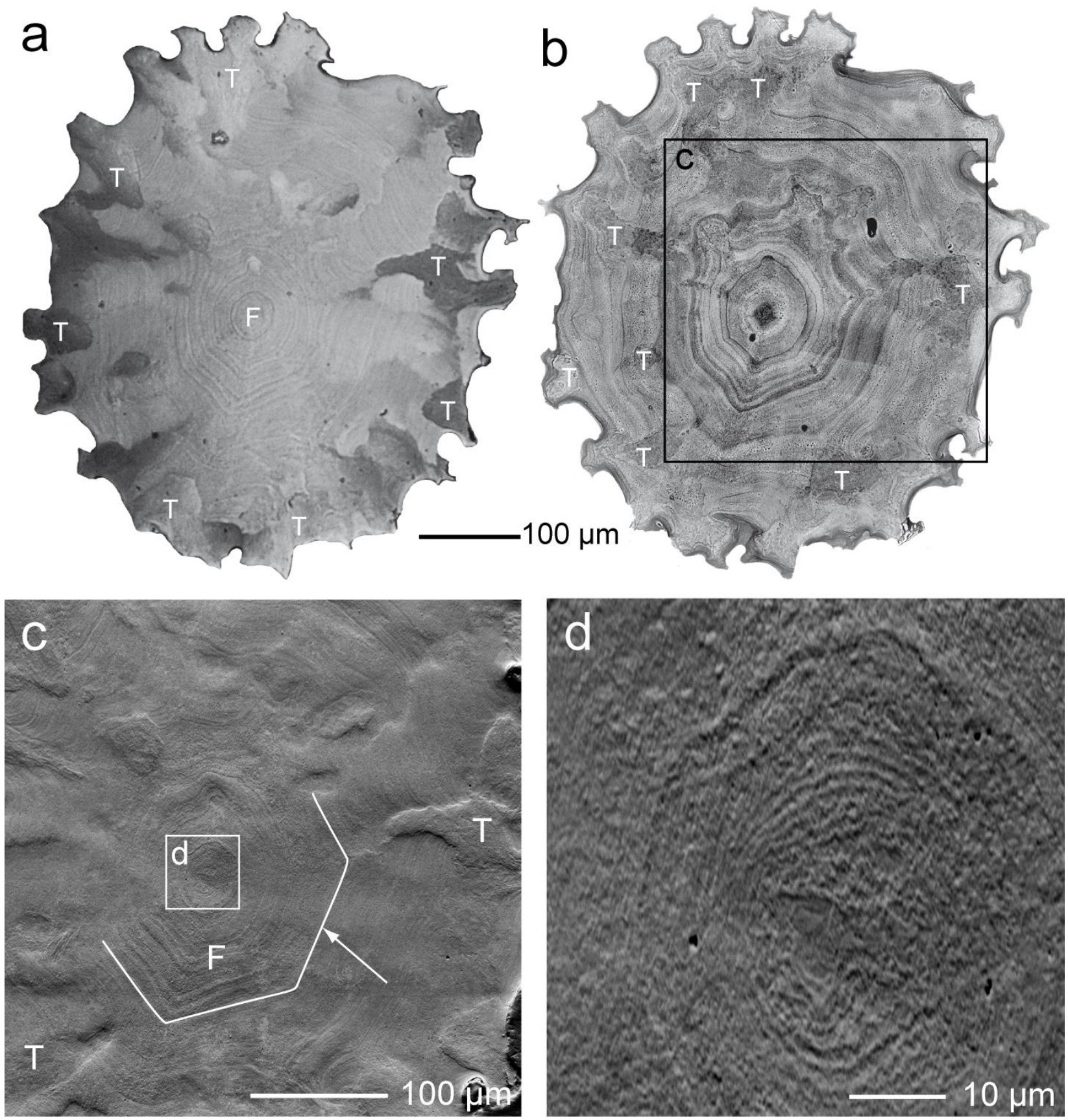

Figure 8: Transverse section of a large Sinularia sclerite (section \#1). a: polarizing microscope image emphasizing crystallographic orientation contrast. b: mosaic of BSE-SEM images showing compositional contrast (mostly variations of magnesium content). c: SEM forward-scattered electron (FSD) image of a portion of $\mathbf{a}$ and $\mathbf{b}$ emphasizing topographical contrast. $\mathbf{d}$ : enlargement of c. F: frame, T: tubercle. 


Figure 9: EBSD study of a large sclerite (transverse section \#1 - Fig. 8). a: Polarizing microscope image of the analyzed sample. b: EBSD map, grid $197 \times 221$, step $3 \mu \mathrm{m}$. c: stereographic projections of $\{001\}$ and $\{1-10\}$. d: density plots of the stereographic projections. The white dot is the trace of the sclerite long axis and the white arrows indicate a rotation of about $22^{\circ}$ with respect to this axis. T: tubercle, $\mathrm{R}, \mathrm{G}$, and $\mathrm{B}$ : red, green, and blue sectors. The three red, green and blue solid meridian lines are traces of the $\{001\}$ plane of the red, green and blue sectors, respectively.
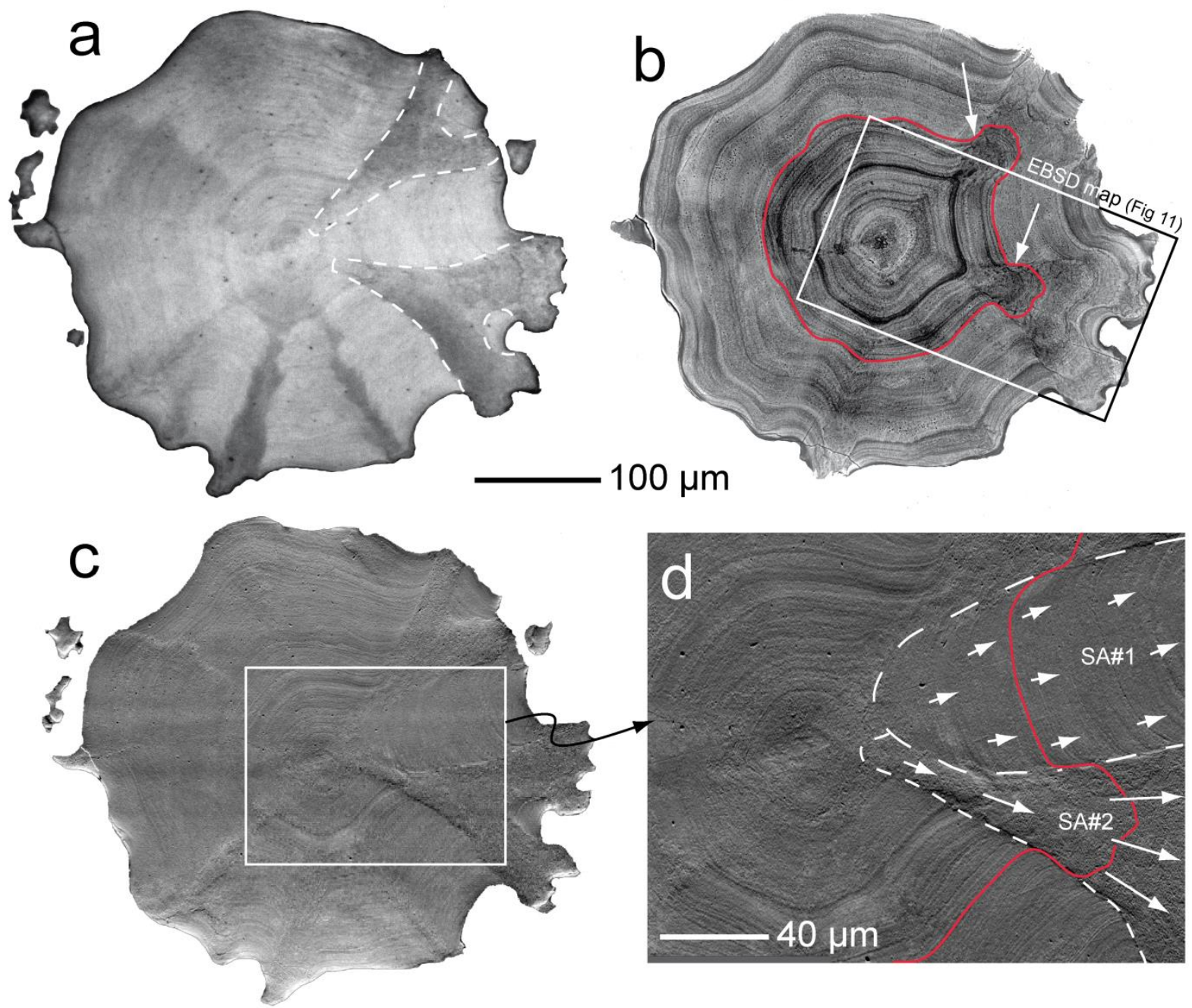

Figure 10: Transverse section of a large sclerite (section \#2). a: polarizing microscope image emphasizing the relative positions of the frame and the tubercles. Two tubercles have been contoured with white dashed lines. b: SEM-BSE mosaic showing growth rings marked by oscillatory zoning in magnesium. The red line marks a paleo growth front. c: SEM-FSE image emphasizing topographic contrast between tubercles and frame. d: detail of $\mathbf{c}$. The white dashed lines delimit selected areas (SA) \#1 and \#2 discussed in the text. White vectors are indicative of the crystallite $c$ axis tilt direction and intensity in SA\#1 and \#2. The red line is the same paleo growth front as in $\mathbf{b}$. Same scale for $\mathbf{a}, \mathbf{b}$, and $\mathbf{c}$. 
Topographic contrast is attributed to contrasting response to polishing of the frame and the tubercles, itself due to difference of crystallographic orientations between the two domains (as will be confirmed below). These SEM FSD images also show that the tubercles are deeply rooted and start growing 20 to $40 \mu \mathrm{m}$ away from the sclerite center (Fig. 10d). The SEM BSE mosaic of Fig. 10b emphasizes the chemical contrasts between growth rings; it allows the observation of continuous concentric growth rings, leaving no doubt on the presence of layering within the tubercles (even if this layering is neither observed on SEM-FSD nor polarizing microscope images (Figs. 10a and c). The EBSD map of the area covering both the inner frame and one of the most obvious rooted tubercle has been collected at relatively high spatial resolution ( $1 \mu \mathrm{m}$ step interval) (Figs. 11a and b). As already determined, the crystallites in the frame are close to perpendicular to the plane of the map, at an angle of about $\sim 22^{\circ}$ with respect to the central axis of the sclerite, and three orientations prevail (Fig. 11c). The difference of intensity of the three spots (white arrows in Fig. 11c) comes from the fact that the EBSD map (Fig. 11b) is off centered with respect to the main sclerite axis. Figure $11 \mathrm{~d}$ is the same map than $11 \mathrm{~b}$, except that the color code has been chosen to enhance the three sectors (blue, green and red). The hexagonal prisms (insets in Fig. 11d) are representative of the crystallographic orientation in each sector (on this map, rooted tubercles are shown in black). These observations confirm the conclusions reached earlier for the frame (Fig. 9). A portion of the frame, comprised between two tubercles, has been selected to better characterize the pattern of crystallite orientations within a frame sector (selected area SA\#1 in Figs. 11a and d). The $\{001\}$ pole density figure (Fig. 11e) confirms that the crystallites are similarly oriented at an angle of about $22^{\circ}$ from the long sclerite axis. This tilt remains remarkably constant within the selected area and does not seem to increase from the center to the rim of the sclerite. Other crystallographic directions $(\{1-10\}$ and $\{104\})$ point out the remarkable similarity of crystallite orientations in the selected area (Fig. 11e). 

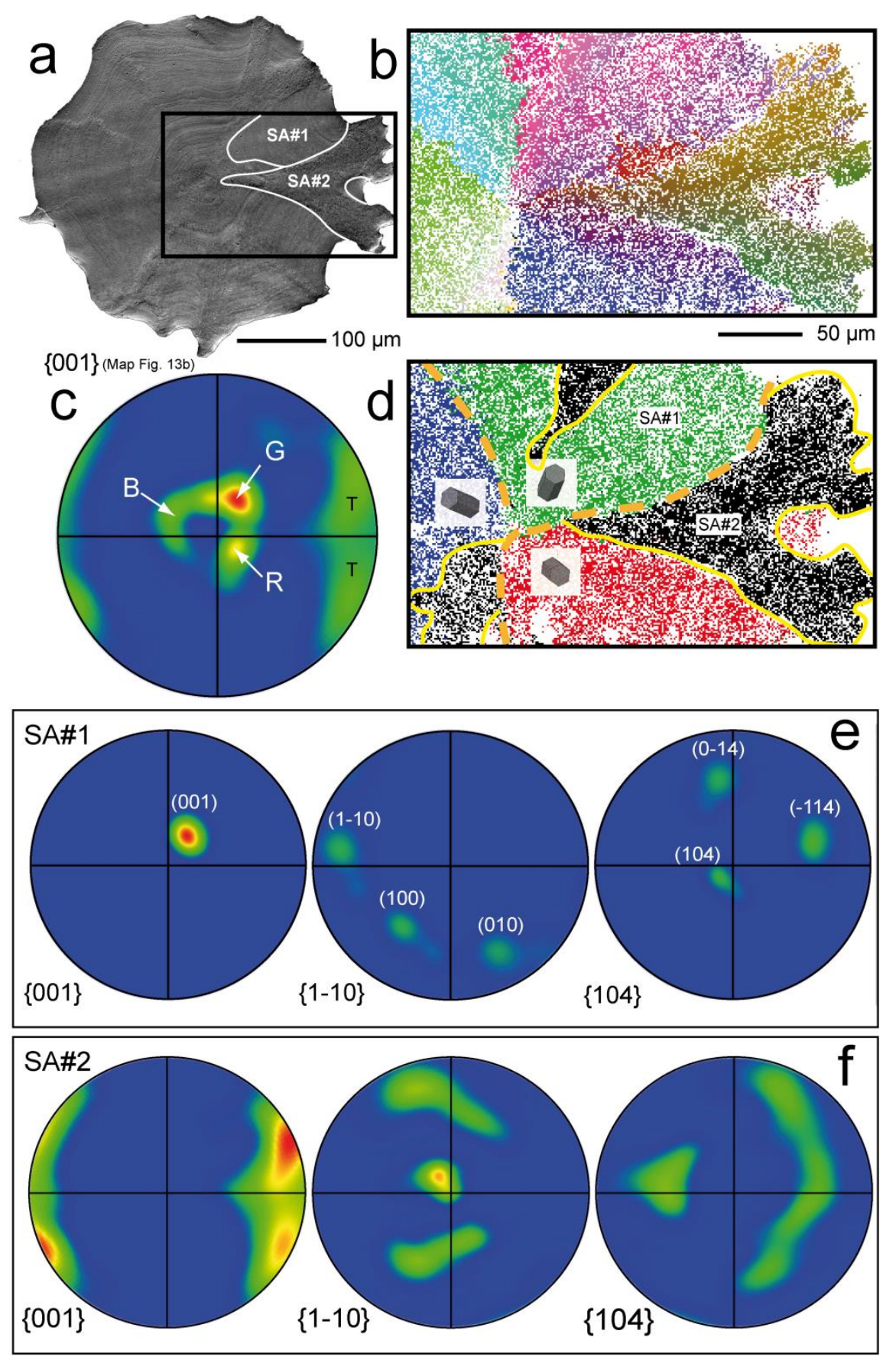

Figure 11: EBSD study of a second sclerite transverse section (\#2) with emphasis on the tubercle. a: position of the EBSD map on the transverse section (see also Fig. 10). b: EBSD map (raw data). c: $\{001\}$ stereographic projection (density plot) for the entire map. d: Same map as in b with a RGB color code selected to emphasize the difference between the three frame sectors (separated by orange heavy dashed lines). The hexagonal prisms are indicative of the mean crystallographic direction in each sector. Tubercles are shown in black. Locations of the selected areas (SA) discussed in the text are shown in a and $\mathbf{d}$. e and f: density plots of $\{001\},\{1-10\}$ and $\{104\}$ in SA\#1 and SA\#2, respectively. 
Concerning the relative orientation between crystallite and growth surface, the SEM-BSE and SEM FSD images (Fig. 10b and d) show that the growth rings are slightly concave towards the rim in SA\#1, and that the curvature increases as the growth front gets closer to the rooted tubercle (Fig. 10b white arrows). In the tubercle itself, the curvature of the growth front reverts to become essentially convex. Thus, at the interface between the frame and the tubercle (Fig. 10b, white arrows), the growth front changes from nearly perpendicular to nearly parallel to sclerite radius. A detailed observation of the EBSD data within SA\#1 shows that the crystallite tilt direction remains approximately parallel to sclerite radius, even when the orientation of the growth front changes from perpendicular to parallel to sclerite radius. Thus, crystallite tilting directions are not necessarily perpendicular to growth fronts; they are in general close to perpendicular to it, but they can be locally at low angle or even parallel to the growth front at the frame/tubercle interface. A schematic view of the relationships between crystallite orientation and growth front in SA\#1 is shown in Fig. 10d with white arrows whose length and direction represent the tilt intensity and direction of the crystallite $c$ axes, respectively.

The area covering a single rooted tubercle has been selected on the EBSD map (SA\#2 Figs. 11a and d). The corresponding $\{001\}$ pole density figure displays a remarkable plume pattern (Fig. 11f), quite different from what is observed in the frame. In the tubercle, the crystallite $c$ axes rapidly tilt with respect to the central long axis of the sclerite from about $25^{\circ}$ near the core, to $90^{\circ}$ to the rim. In other words, away from the sclerite center, the crystallite $c$ axes lie on the plane of the EBSD map (Fig. 11b), on the primitive circle (Fig. 11f). On the other hand, in the plane of the map, the $c$ axis directions vary and depart from the plume main axis within the range $+/-45^{\circ}$; this is shown in the $\{001\}$ pole density figure (Fig. 11f). Figures $12 \mathrm{a}$ and $b$ are two differently color-coded EBSD maps of the same tubercle. 

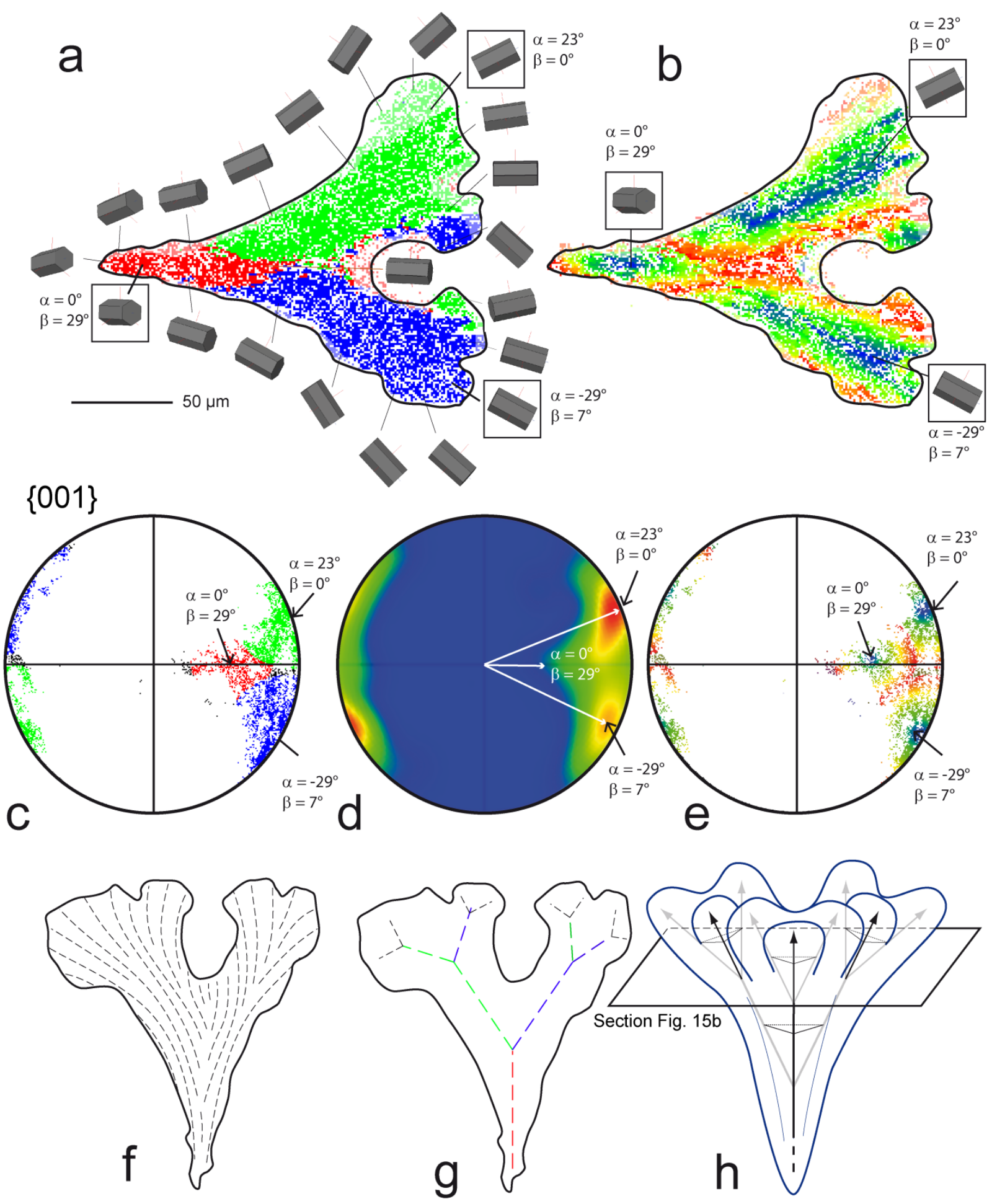
Figure 12: EBSD study of a tubercle (SA\#2 in Fig. 11). a: color code selected to emphasize the three main crystallographic orientations in the tubercle (red: domain with $\alpha=0^{\circ} \beta=29^{\circ}$; green: $\alpha=23^{\circ} \beta=0^{\circ}$; blue: $\alpha=-29^{\circ} \beta=7^{\circ}$ (significance of the $\alpha$ and $\beta$ angles in Fig. $7 \mathrm{c}-$ see also the three hexagonal prisms in the insets; tolerated deviation: $+/-20^{\circ}$ in each domain). The hexagonal prisms shown around the tubercle illustrate the progressive change of crystallite orientations. $\mathbf{b}$ : Color code selected to emphasize the progressive crystallographic $c$ axis deviations from the above mentioned $\alpha$ and $\beta$ in each tubercle domain. Deviations increase in the order blue, green, yellow, and red. c: stereographic projections of the $\{001\}$ planes of the tubercle (same color code as in a). d: density plot of $\{001\}$ with indication of the $\alpha$ and $\beta$ angles. e: stereographic projections of $\{001\}$ (same color code as in $\mathbf{b}$ ). f: representation of the $c$ axis orientations in the tubercle. g: idealized fractal pattern of the crystallite orientations. $\mathbf{h}$ : 3D representation of the shape of a tubercle as a self-similar inverse trigonal pyramid.

Figure 12a emphasizes the presence of three distinct domains indicated in red, green and blue; the mean $c$ axis orientation are $\alpha=0^{\circ} \beta=29^{\circ}, \alpha=23^{\circ} \beta=0^{\circ}$, and $\alpha=-29^{\circ} \beta=7^{\circ}$ in the red, green, and blue domains, respectively [the significance of the $\alpha$ and $\beta$ angles is given in Fig. 7c (see also the framed hexagonal prisms in Fig. 12a)]. A euler angle deviation of $+/-20^{\circ}$ with respect to these mean values has been assigned in each domain. Figure $12 \mathrm{c}$ is a stereographic projection of the $\{001\}$ planes with the same color code than in Fig. 12a; it gives an idea of the dispersion of orientations within each sector. Figure $12 \mathrm{~b}$ is another representation of the same tubercle that emphasizes the progressive change of orientation within the $R, G, B$ domains. Blue pixels correspond to crystallites $c$ axis orientations close to the angular values assigned in each domain (see the three hexagonal prisms in Fig. 12b). As the orientation departs from these values, the color progressively changes from blue, green, yellow and red. Figure $12 \mathrm{e}$ is a stereographic projection of the $\{001\}$ planes with the same color code than Fig. 12b. The hexagonal prisms surrounding the tubercle in Fig. 12a give an idea of the progressive changes of crystallite orientations in the tubercle. A more complete scheme of crystallite $c$ axis orientations in the plane of the map is provided in Fig. 12f (dashed lines). The multilevel branched (or fractal) pattern of 
the tubercles is schematized in Fig. 12g. The 3D organization and morphology of the tubercle (Fig. 12h) will be discussed later.

\section{Discussion}

\subsection{The crystallographic structure of large Sinularia sclerites: a summary}

At this stage, the different structural units of the large sclerites are identified. At the center, protosclerites are interpreted as nuclei around which large sclerites grow. Concerning the frame, Fig. $13 \mathrm{a}$ is a representation of the crystallite $c$ axes orientations in a longitudinal section of a large sclerite with the corresponding simulated density plot (Fig. 13b). The crystallite $c$ axes are rarely strictly perpendicular or parallel to growth surfaces; most commonly, the angle between crystallite orientation and surface front is close to $\sim 20^{\circ}$. This conceptual figure may suggest that the long axis of the sclerite is a rotational axis. However, as demonstrated earlier, transverse section indicate that three crystallite orientations prevail (Fig. 9d). The $c$ axis orientations in transverse sections are schematized in Fig. 13c, the crystallite $c$ axes are close to perpendicular to the figure plane and thus, can no longer be represented by small rods (as in Fig. 13a), they have to be represented by vectors. The direction and length of the vectors correspond respectively to the tilting direction and tilting magnitude of the $c$ axes (see ${ }^{8 b}$ for a similar type of representation). Crystallite orientations in Fig. 13c are consistent with the density plot shown in Fig. 13d, with three maxima at respective angles of $120^{\circ}$, a tilt of about $20^{\circ}$ with respect to the long sclerite axis, and the near absence of orientation strictly parallel to this axis. Fig. 13e is a conceptual 3D wire model of crystallite orientations in the large sclerite frame emphasizing the

presence of a center of symmetry, and the opposite trihedral arrangement of the crystallite orientations. 

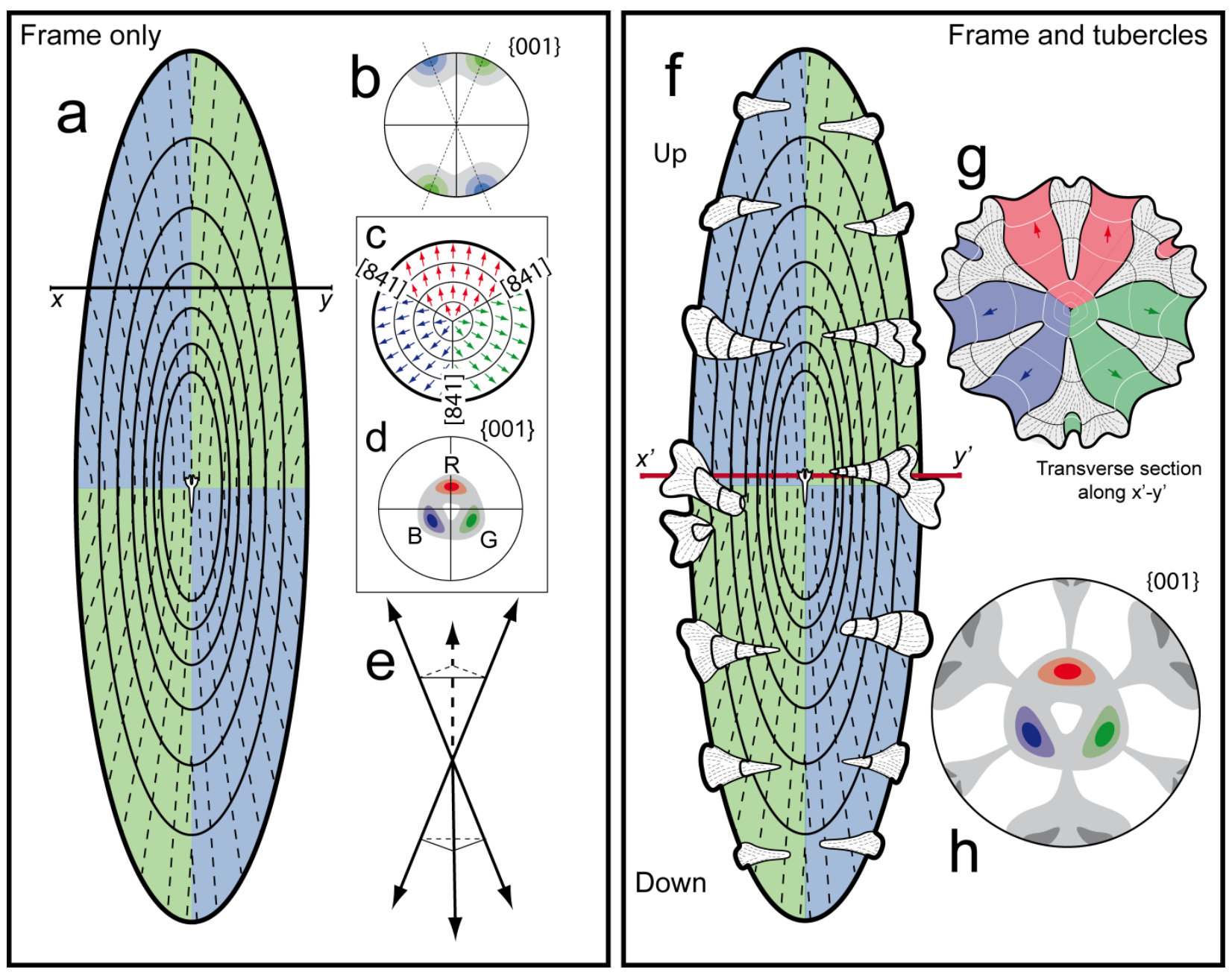

Figure 13: Structural features of large Sinularia sclerites: a summary. a: longitudinal section without tubercles. Dashes represent crystallite $c$ axis orientations, and concentric ellipses the successive growth rings. Note the presence of a protosclerite that served as a nucleus for further growth. b: density plot of the $c$ axis orientations in a (compare to Fig. 7d). c: transverse section along $x-y$ in (a); $c$ axes are now close to perpendicular to the figure plane, the vector directions and lengths represent the tilting direction and magnitude of the $c$ axes, respectively. Concentric circles represent successive growth rings. d: density plot of c, compare to Fig. 9d. e: 3D representation of major crystallite orientations in a large sclerite frame; it emphasizes the trigonal symmetry of the orientation pattern. f: longitudinal section with the tubercles. Contourned successive growth rings are also indicated. $\mathbf{g}$ : transverse section with tubercles along $x^{\prime}-y^{\prime}$ in (f); in the frame, $c$ axes are close to perpendicular to the figure plane, the vector directions and lengths represent the tilting direction and magnitude of the $c$ axes; in the tubercles, crystallite $c$ axis orientations are close to parallel to the figure plane and are represented as dashes. Contourned concentric circles represent successive growth rings; compare to Fig. 8a and b. h: $\{001\}$ stereographic projection (density plot) of $\mathbf{g}$. 
Perpendicularly to the long sclerite axis, deeply rooted tubercles develop with crystalline fibers and $c$ axis crystallites close to parallel to the tubercle axis. Inside the tubercle, in the plane normal to the long sclerite axis, the $c$ axis crystallite orientations diverge progressively from the successive axes of the branched tubercle (Figs 12f and g). The crystallographic structure of a complete sclerite (frame and tubercles) is schematized in Fig. 13f and g. The corresponding density plot of the $\{001\}$ stereographic projections is shown in Fig. 13h.

To summarize, in contrast with small sclerites, large Sinularia sclerites are complex hierarchical mesocrystalline structures, that can be referred to as 'poly mesocrystalline'. These sclerites correspond to type (4) defined by Schmidt ${ }^{6}$.

\subsection{The 3-D shape of the tubercles and generalization of the trigonal model}

Different observations suggest that deeply rooted tubercles have a trigonal inverse pyramid (or tripod) shape in 3D. Indeed, most Sinularia proto- and small sclerites display tripod-looking, dart morphologies. In addition, the crystallites in the tubercles progressively rotate around the three equivalent $\boldsymbol{a}$ axes of the calcite hexagonal unit cell. This feature has already been observed in the red coral sclerites ${ }^{8}$ and it has been demonstrated that this organization translated into a tripod morphology. It is likely that the same relationship between crystallite orientation and morphology exists in the Sinularia tubercle. The resulting idealized 3D morphology of a Sinularia tubercle is shown in Fig. 12h. This figure is consistent with the multilevel branched structure discussed above, meaning that each branch subdivides into three new branches at constant angle, in a process repeated three to four times to form a tree-like structure with self-similar character (Fig. $12 \mathrm{~g})$. As seen above, the surface of the Sinularia sclerite is studded with more or less regularly spaced jagged tubercles $(\sim 100 \mu \mathrm{m}$ between tubercle axes) creating concave space $(\sim 20-50 \mu \mathrm{m}$ 
wide) between them (Fig. 3c). As a working hypothesis, following the idea developed for $C$. rubrum that the skeleton shape is influenced by organs (gastrodermal canals or polyps) at its surface $^{14}$, the surface morphology of Sinularia sclerites could be constrained by the shape of cells or vesicles constituting the mineralizing epithelium at its surface. The idea of confinement of inorganic deposition within organized tissues is in agreement with the general theory on growth and form of biominerals developed by Mann ${ }^{1}$. The fractal nature of deeply rooted tubercles has the advantage of increasing the number of tubercles at the surface of the sclerite as the diameter increases, keeping the number of tubercles per surface unit, and the distance between them, constant at various growth stages. The alternation and regular spacing of concave spaces and convex tubercles could be a way to accommodate external molding constraints. The biological function of such a rugged surface could be to allow a mechanical coupling between the sclerite and the organic tissues; a similar function could be attributed to microprotuberances at the red coral skeleton surface.

The trigonal inverse pyramid is a fundamental structure of Sinularia sclerites, well recognized in deeply rooted tubercles but also observed in the axial frame. Indeed, the axial frame can be described as a centrosymmetric mesocrystalline structure with two tripods in opposite trihedral arrangement (Fig. 13e), similar to red coral sclerites. The fact that the large ovoid Sinularia sclerites do not have the dumbbell shape of $C$. rubrum sclerites is attributed to deeply rooted tubercles (transverse tripods) that superimpose upon the sclerite frame. An important consequence of the presence of numerous deeply rooted tubercles at the sclerite surface is that in these domains, crystallite $c$ axes remain perpendicular to the growth surface which is not the case in the frame domains (see Fig. 13f). 


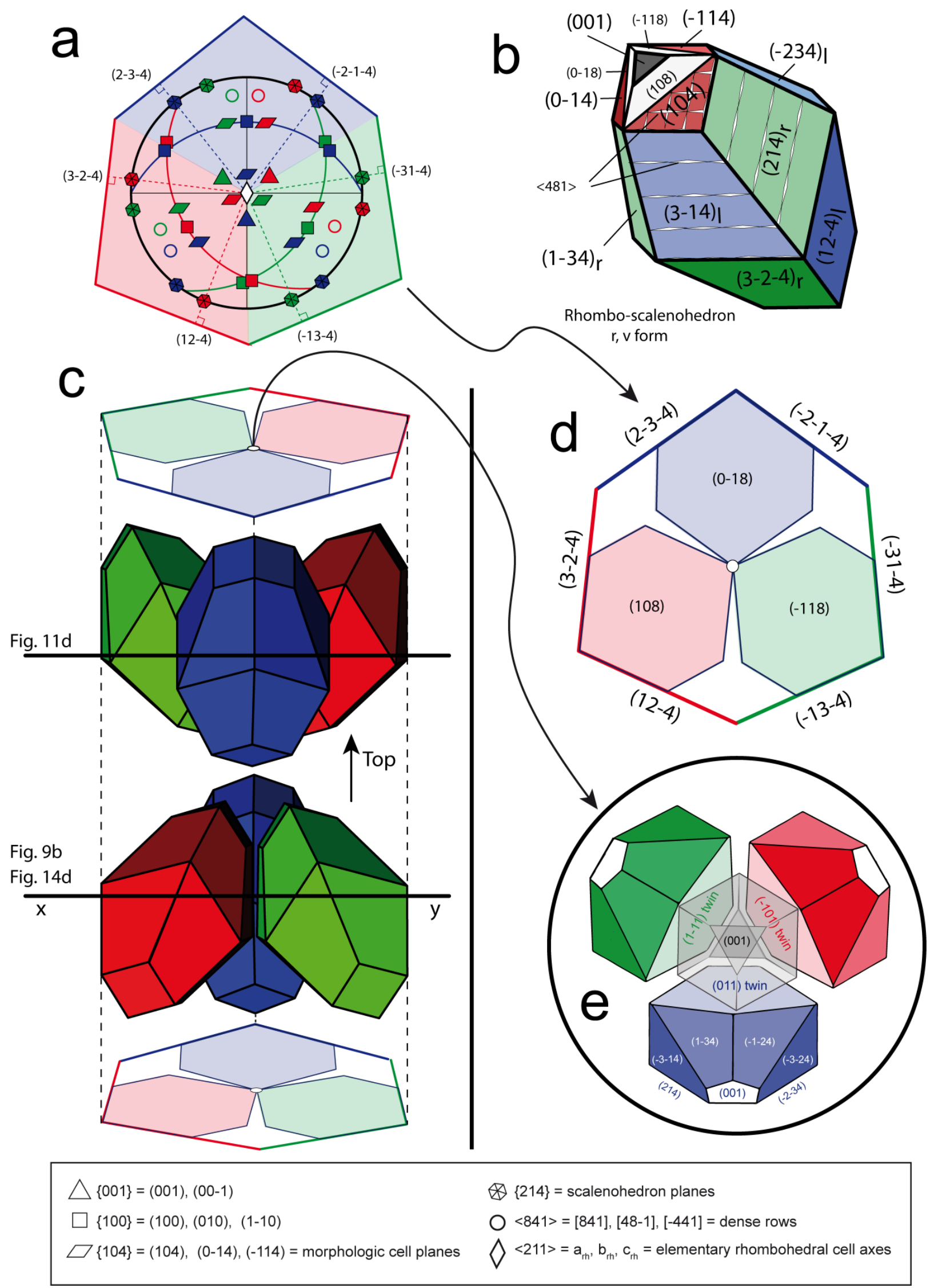


Figure 14: Crystallographic pattern of the frame: a model. a: Synthetic stereographic projection of the main planes and dense rows of the RGB sectors displayed in Fig. SI\#5 (see also Fig. 9d). The red, green and blue great circles correspond to the R, G, B $\{001\}$ planes, respectively. Some indexes have been omitted for clarity, they are reported in Fig. SI\#5. The $\{214\}$ scalenohedron planes are shown as hexagons: their poles plot on the primitive circle, indicationg that they are parallel to the long sclerite axis. Their traces are plotted outside the primitive circle, in their respective R, G, B colors, to outline the possible relationship between the hexagonal morphology of the core (Fig. 8a) and the hexagonal crystallographic organization. b: theoretical rhombo-scalenohedron of calcite with its indexed faces and dense rows. c: 3D idealized crystallographic organization of the frame with scalenohedra as basic units. d: section of the 3D model (along $x-y$ in c) showing the relationships between the scalenohedron faces and the pseudo-hexagonal frame shape at the sclerite center. e: a model for the initiation of the three sectors in the frame by mesotwinning on a protosclerite.

\subsection{A Sinularia sclerite crystallographic model}

Previous studies have shown that principles of crystallography can be used to study the imperfect but statistically ordered crystalline superstructures in biominerals ${ }^{8 a}, 15$. The crystallographic model presented below is based on these premises and should be considered accordingly. This model will address the following question: is there a crystallographic scheme behind the different sets of crystallite orientations in the frame and the tubercles, and at their interface? Are crystallite orientation changes associated with progressive rotation or mesotwinning? And finally, do crystallite misorientations tend to a maximum or not?

Crystallographic scheme in the sclerite frame: Data from the three frame sectors labelled R, G and B in Fig. 9d have been extracted from the whole dataset, slightly rotated to set the long sclerite axis exactly perpendicular to the primitive circle, and plotted on $\{001\},\{1-10\},\{104\}$ and $<841>$ stereographic projections (density plots) (Fig. SI\#5). For clarity, the R, G and B domains have been treated and indexed separately (Figs SI\#5 a to c). All sectors display remarkable crystallographic orientations with limited misorientation. In Figs SI\#5d, the pole figures of all sectors have been put on top of each other. The $\{1-10\}$, and $<841\rangle$ pole figures show exact coincidence of the R and G, R and B, and G and B spots. All these relationships can be synthesized in a single pole figure (Fig. 14a), each spot from Fig. SI\#5 being represented by a 
single symbol (compare Fig. 14a and Fig. SI\#5d). It is important to recall here that $\{001\},\{1$ $10\}$ are important family planes of the calcite elementary and hexagonal cells. $\{104\}$, and $\{214\}$ are faces that belong to two of the most common habits found on natural inorganic calcite: the morphological rhombohedron ["Iceland spar" crystal designated $r\{104\}$ ] and the chiral scalenohedron [“dog-tooth" crystal designated $v\{214\}]$. The two families of faces $\{104\}$ and $\{214\}$ (or $\{234\}$ ) share the property of being built from the same periodic $\mathrm{Ca}^{2+} \mathrm{CO}_{3}{ }^{2-}$ chains (the $<841>$ rows), the strongest $\mathrm{Ca}^{2+} \mathrm{CO}_{3}{ }^{2-}$ chain in calcite ${ }^{8 \mathrm{a}, 16}$, electrically neutral, and without dipole moment. A first remarkable feature of Fig. 14a is that the $\{214\}$ planes of the three sectors plot exacly on the primitive circle of the stereographic projection. As stated earlier, these are characteristic planes of the scalenohedron, which means that in each frame sector, two scalenohedron planes are parallel to the long sclerite axis. The traces of these planes (usually drawn inside the primitive circle) have been projected outside the primitive circle in Fig. 14a for the three R,G, B sectors. All these traces define together an irregular hexagon that is reminiscent of the geometrical features observed at the center of the sclerite sections (see Figs. 8c and 10). Thus, the traces of the hexagons in the sclerite frame could correspond to traces of $\{214\}$ faces that contain the $<841>$ densest rows (as can be verified on Fig. 14a). Note that these $\{214\}$ and $\{234\}$ mirror related faces of the calcite scalenohedron, are half as dense as $\{104\}$ faces (Fig. 14b, and also Fig. 3f in ${ }^{8 a}$ ). They are chiral ${ }^{16-17}$, nonpolar and asymmetric with a stepped face character. Another singularity to emphasize in Fig. 14a concerns the center of the stereographic projection. The three sectors $(\mathrm{R}, \mathrm{G}, \mathrm{B})$ share a common axis located at the center of the stereographic projection. This axis correspond to <211> family rows matching the three $a_{r h}, b_{\mathrm{rh}}$, and $c_{r h}$ axes of the elementary rhombohedral calcite cell. The $\{108\}$ planes in each sector are perpendicular to these axes and lie in a same plane perpendicular to the long sclerite axis as illustrated in Fig. 14d. Thus, although crystallographic directions are different in each sector, all 
sectors have in common an axis of the calcite elementary cell and a $\{108\}$ plane. Now, is the transition of crystallographic orientation from a sector to another progressive or sudden? A progressive change of orientation involves a process of ordered misorientations; on the contrary, a sudden change may involve a process of mesotwinning, an analog of twinning with defects ${ }^{8 \mathrm{a}}$. Both processes have been defined and observed in the red coral sclerites ${ }^{8}$. The near absence of $c$ axis crystallite orientation parallel to the long sclerite axis (Fig. 9d) is in favor of sudden change of crystallite orientations. Furthermore, the poles of the three sector interfaces coincide within $5^{\circ}$ with scalenohedron faces of the two sectors involved in the interface (see Fig. 14a). The juxtaposition of $\{214\}$ faces between two sectors favors again the idea of $\{214\}$ mesotwinning rather than progressive misorientations. It must be noted that twinning along $\{214\}$ is not classic, contrary to twinning along $\{001\},\{104\},\{012\}$ and $\{018\}$; however, this type of (214) relationship has implicitly been recognized in the red coral skeleton (Fig. $2 \mathrm{c}-\mathrm{d}$ in ${ }^{8 \mathrm{a}}$ ). Could $\{214\}$ be a new mesotwin plane in biominerals? Further observations are required to answer this question. This conclusion raises the question of the initiation of the three sectors. The observed presence of a protosclerite at the center a large sclerite lead to the possiblility of a nucleus, elongated along its $c$ axis, a direction that will coincide later with the long sclerite axis. Mesotwinning on the (011), (-11-1) and (-101) planes of the protosclerite may represent the initiation of the three sectors observed in the large sclerite. This configuration illustrated in Fig. $14 \mathrm{e}$ would explain the presence of a symmetry center and the opposite trihedral arrangement of crystallographic orientations in the large sclerite frame as schematized in Fig. 14c. Figure 14d links the three-D model (Fig. 14c) to the original observations arranged in Fig. 14a. It must be emphasized that despite the remarkable crystallographic relationships, there is almost no interpretation input in Fig. 14a which is a mere plot of relationships observed in Fig. SI\#5. Similar relationships have been observed in other EBSD sections but are not presented here for 
the sake of conciseness. Figures $14 \mathrm{c}$ and $14 \mathrm{e}$ are more interpretative than Fig. 14a but they remain consistent with the actual data.

Crystallographic scheme in the tubercles: To model the crystallite orientations in the tubercles, the three major crystallite orientations from the tubercle shown in Fig. 12a have been reprocessed and plotted in the $\{001\},\{104\},\{1-10\}$, and <841> pole figures (Fig. 15a). To facilitate the discussion, all data have been rotated to set the tubercle axis perpendicular to the plane of observation (which explains the difference between the $\{001\}$ projections of Fig. 12c and 15a). The crow's foot features observed in the $\{001\}$ and $\{104\}$ pole figures emphasize the progressive rotation of the crystallites primarily around three axes. These three axes can be unambiguously identified in the $\{1-10\}$ pole figure as the three equivalent $\boldsymbol{a}$ axes of the hexagonal unit cell of calcite (i.e. [1-10], [100], and [010]), orthogonal to the $\langle 841\rangle$ directions and to the $c$ axes. In a previous article, numerical simulations using the CaRIne Crystallography software clarified the 3D misorientation pattern in the red coral sclerite (Figs. 11 and 12 in ${ }^{8 b}$ ). The simulated pattern of ordered misorientations replicated in Fig. 15b and its corresponding pole figure (Fig. 15c) reproduce the crystallographic pattern of the Sinularia tubercle, just as in the red coral sclerite case ${ }^{8 b}$. In Sinularia, the imperfections and departure from the theoretical case can be attributed to the fractal character of the crystallographic organization in the tubercle (see Fig. $12 \mathrm{~g}$ ). The model from Fig. $15 \mathrm{~b}$ is consistent with the low degree of misorientation in the central axis of the tubercle, the progressive change of direction and the progressive inversion of direction within a pod and between different pods. This pattern combines the existence of well-identified domains of misorientation in the tubercle with a progressive transition between them. 


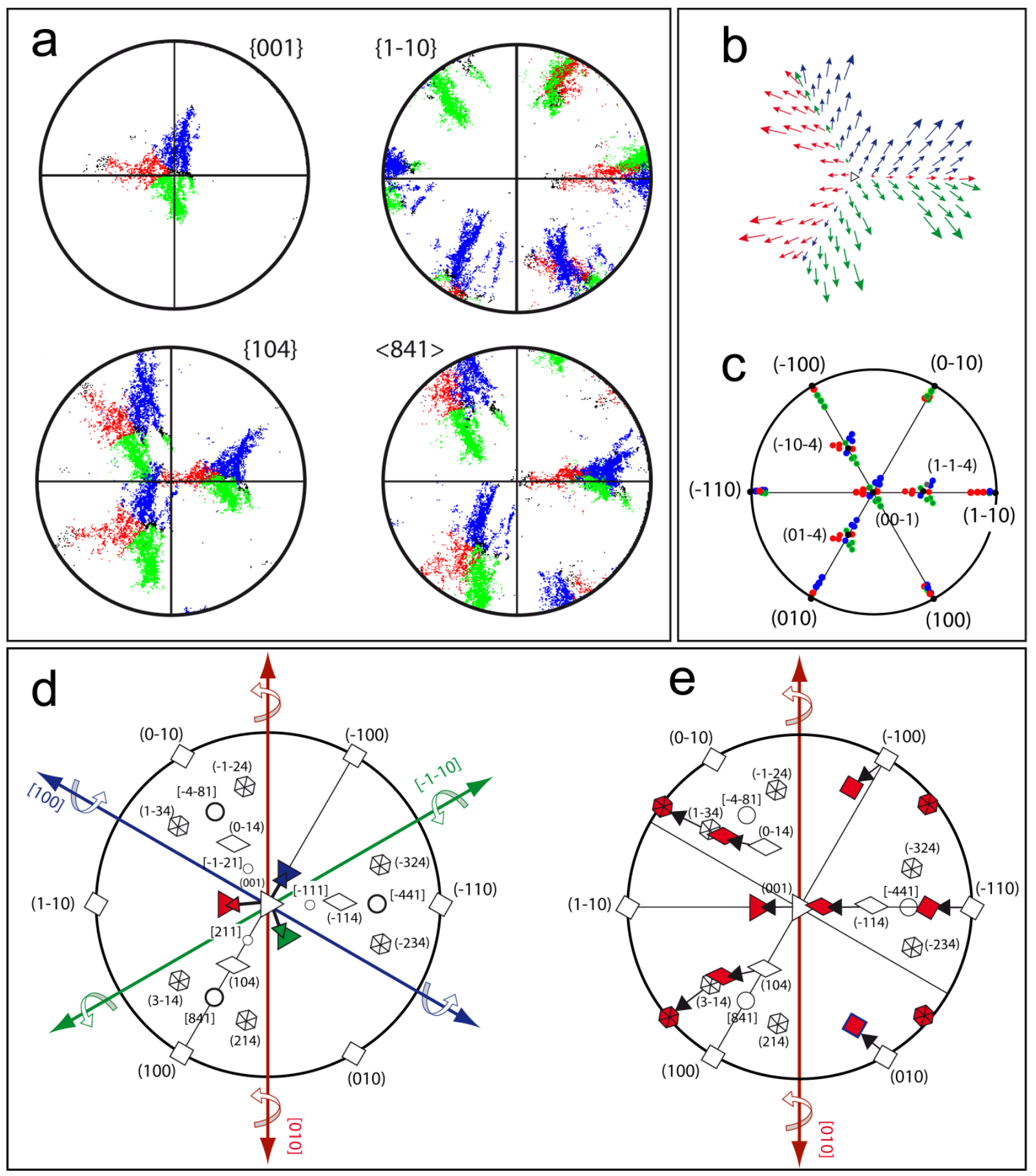


Figure 15: Crystallographic pattern of a tubercle: a model. a: stereographic projections of the $\{001\},\{1-10\},\{104\}$ planes and $<841>$ dense rows of the tubercle (SA\#2) of the transverse section shown in Fig. 11 (red, green, and blue domains as in Fig. 12a). The data have been rotated to set the long tubercle axis perpendicular to the primitive circle; hence the difference between the projections of the $\{001\}$ planes in Fig. 12c and 15a. b: Simulated misorientation pattern in a section perpendicular to a tubercle (see Fig. 12h for a schematic location of the section). The direction and length of the vectors represent the $c$ axis tilt azimuth and magnitude, respectively. The RGB color code is related to the three sectors defined in Fig. 15a (see also Fig. 12a). c: stereographic projection of $\{001\},\{104\}$ and $\{1-10\}$ of the simulated pattern in Fig. $15 \mathrm{~b}$ (compare to the real case in Fig. 15a). d and e: synthetic stereographic projections of the tubercle related to Fig. SI\#6. See text for explanation.

In order to underline similarities and differences between the inverse trigonal pyramid arrangement in the frame and the tubercle, a crystallographic analysis similar to that of the frame (Figs. 14 and SI\#5) has been carried out on the tubercle. In Fig. SI\#6, the density plots of the pole figures of $\{001\},\{100\},\{104\}$, and $<841>$ of the R, G, B domains of the tubercle from Fig. 15a have been reported and indexed separately. The similarity of patterns in the tubercle and the frame is remarkable (compare Figs SI\#5 and SI\#6). To interpret these data, the empty symbols in Fig. 15d represent planes and rows of an ideal calcite crystal with its $c$ axis perpendicular to the primitive circle. The three equivalent $\boldsymbol{a}$ axes of the hexagonal unit cell of this crystallite (i.e. [-110], [100], and [010]) are also shown. If this crystal rotates around the [010] axis by $26^{\circ}$, the position of the (001) plane changes from the empty to the red triangle. Similar rotations around the [-1-10] and [100] axes generate the green and blue triangles, respectively. A large set of planes and rows is shown for the case of the rotation of the original crystal around the [010] axis (Fig. 15e - empty to red symbols). This simulated stereographic projection is in agreement with the features observed in the tubercle for the red sector (Fig. SI\#6). Rotations around [-1-10] and [100] would generate configurations consistent with the green and blue sectors, respectively; they 
are not shown in Fig. 15e for the sake of clarity. Summarizing, the crystallographic features of the tubercle are consistent with a set of crystallites that would progressively rotate around three preferential axes. During the previous presentation of the crystallographic features of the tubercle, a maximum rotation angle of $22^{\circ}$ has been mentioned. The difference between $22^{\circ}$ and $26^{\circ}$ [determined in this model (Fig. 15e) to match the tubercle data from Fig. SI\#6] can be ascribed to the fractal nature of the crystallographic pattern in the tubercle that progressively increases the value of this angle (see Fig. 12g).

The synthetic stereographic projections of the frame and the tubercle (Figs 14a and 15e), are remarkably similar. It implies that the trigonal inverse pyramids observed in the frame and the tubercles tend towards similar crystallographic configurations. However, one of the pyramids is achieved through a sudden change of crystallographic orientations (in the frame), possibly through a mesotwinning process, while the other (in the tubercle) is reached through progressive misorientations of the crystallites. In both cases, a trigonal geometry has emerged, inherited (or transposed) from the original trigonal structure of a single crystallite.

Interface between the frame and the tubercle: Two areas have been selected to study the crystallographic relationships at the frame/tubercle interface (SA\#3 and \#4 in Fig. 16a, yellow rectangles). Traces of the interfaces (heavy blue line) and orientations of the crystallites (drawn as hexagonal prisms) on both sides of the interface have been reported on the EBSD map in Fig. 16a. The stereographic projections (density plot) of the frame and tubercle crystallites from the two SAs are shown in Figs $16 \mathrm{~b}$ and $\mathrm{c}$. The $c$ axes of the frame and the tubercle make an angle of about $53^{\circ}$, but both axes lie on a same $\{110\}$ plane. The direction on this plane coincides with the direction of the interface. Thus, crystallites at the interface can be seen as two hexagonal prisms adjoined along $\{110\}$ but not twinned along this face as the orientation of rows in the two $\{110\}$ 
adjoined faces do not coincide. However, other singular crystallographic relationships can be noted between the frame and the tubercle.

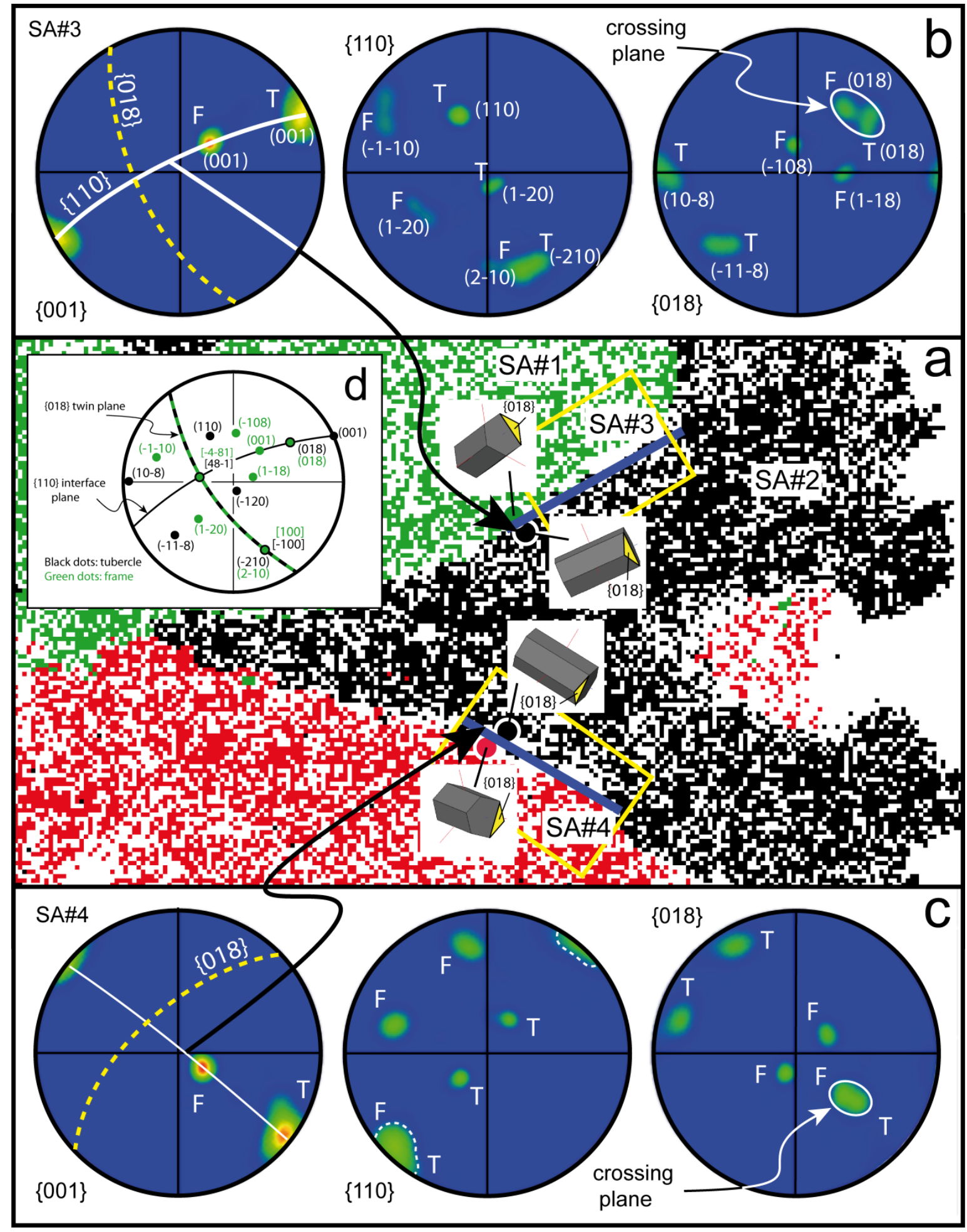


Figure 16: Crystallographic relationships at the interface between tubercle and frame. a: portion of the EBSD map shown in Fig. 11d. Positions of selected areas \#3 and \#4 as in Figs 11a and d. The hexagonal prisms on both sides of the two selected interfaces indicate the orientation of the crystallites. The two heavy blue lines underline the orientation of the interface in SA\#3 and \#4. b and c: stereographic projections (density plot) of $\{001\},\{110\}$, and $\{018\}$ in SA\#3 and \#4, respectively. d: stereographic projection of some characteristic planes and rows in a perfect twinning along (018). Compare to the Sinularia case in Fig. 16b.

For instance, on the $\{001\}$ stereographic projections, the trace of the $\{018\}$ plane is perpendicular to $\{110\}$, the interface plane. As a reminder, twinning among two crystals along a given face can be defined by the apposition of the two faces plus the coincidence of two independent row directions in the two faces. In the theoretical case of $\{018\}$ twinning, [100] and [48-1] are two rows that coincide, as displayed in the stereographic projection in Fig. 16d. Here, the twin plane is shown as a dashed green and black great circle. The comparison of these theoretical relationships to the natural case displayed in Fig. 16b shows stricking similarities. However these relationships cannot be interpreted in terms of $\{018\}$ mesotwinning because the apposition plane is not $\{018\}$ but $\{110\}$. To summarize, tubercle and frame are adjoined along $\{110\}$ and show similar orientations of their $\{018\}$ planes and [48-1] dense rows (see Fig. 16d). The question whether and how these particular crystallographic relationships persist all around the tubercle/frame interface is not still answered.

\subsection{Different stages of sclerite growth}

Structural and chemical markers inside Sinularia sclerites provide constraints to better understand the different stages of sclerite growth. Previous studies indicated that octocoral sclerites form within cells or clusters of cells (scleroblasts); once formed, it is generally assumed that sclerites are expelled from the scleroblast and grow extracellularly [see the cases of Leptogorgia virgulata $^{18}$ and Pseudoplexaura flagellosa $\left.{ }^{10 \mathrm{~b}}\right]$. In these studies, little is said about 
structural differences between intracellular and extracellular growth patterns. Our data show that some protosclerites are enclosed in large sclerites and may act as nuclei around which growth proceeds. The sclerite structure rapidly complexify with the appearance of tubercles perpendicular to the long sclerite axis. It is tempting to connect the change from simple (in the proto and small sclerites) to more complex structure (in large sclerites) to a change of crystallization environment from intra- to extra-cellular. If so, mono-mesocrystalline structures (proto- and small sclerite) could be associated with intra-cellular (or intra-scleroblast) environment while poly-mesocrystalline structures (large sclerite) could be related to extracellular growth. This hypothesis remains to be confirmed.

In agreement with the idea that curved surfaces in biominerals are often signs of the involvement of cellular membranes ${ }^{3}$, microprotuberances could be indicative of the presence of mineralizing tissues (or multicellular vesicles ${ }^{10 \mathrm{~b}, 19}$ ) covering the sclerite. This is in agreement with observations made on Pseudoplexaura flagellosa sclerites $^{10 \mathrm{~b}}$. Concerning the place of the organic matrix in the crystallization process, some authors observed that the first stage of sclerite formation in other species of the octocoral subclass is the deposition of fibrous organic matrix within a vacuole $\mathrm{e}^{20}$. Thus, crystal formation would be associated with organic material $20 \mathrm{a}, 20 \mathrm{c}, 2122$. For some authors, the main organic filaments would subdivide into secondary and tertiary structures and crystallization would proceed in between ${ }^{21}$. In this case, the crystallization would be orchestrated by the organic matrix. The present study does not bring new information on the organic matrix in Sinularia sclerites. Thus, whether the organic matrix organization inside the sclerite predates or is a consequence of the crystalline organization is not known. 


\subsection{Crystallographic tools to achieve complex forms}

The importance of mesocrystals in biominerals: Biominerals are essentially mesocrystalline in nature. The concept of mesocrystal developed by Cölfen and $\mathrm{Mann}^{23}$ (see also ${ }^{24}$ and references therein) and elaborated on the concepts of "particle aggregation" and "nanocrystal superlattices" developed in colloids ${ }^{25}$ apply primarily to superstructures made of nanograins $(<1000 \mathrm{~nm})$. As a new concept, the definition of mesocrystal is still discussed ${ }^{26}:$ one of the latest definitions states that mesocrystals are nanostructured materials with a defined long-range order on the atomic scale, with evidence that the material consists of individual nanoparticle building units ${ }^{27}$. In the present study, mesocrystals are simply defined as 3D superstructures made of sub-micrometer crystallites in crystallographic register. The formulation 'crystallite in crystallographic register' instead of 'similarly oriented crystallites' as used in a previous work of our group ${ }^{8 \mathrm{~b}}$ results from the putative idea that ordered misorientation and mesotwinning initially observed in the red coral and now in Sinularia sclerites could be more common in biomesocrystals than documented so $\mathrm{far}^{28}$. The fact that crystallites are similarly but not identically oriented in mesocrystalline structures has been underlined as soon as the mesocrystal concept emerged ${ }^{24 b}$, 29 . This fact raises the question whether a maximum degree of misorientation should be stated in the definition of mesocrystal. The study of the octocoral sclerites provide an ambiguous answer to this question. It could be 'Yes' if we consider that a maximum value for misorientations of $+/-22^{\circ}$ is often observed in each branch of a tubercle (the present study), in the red coral dumbbell sclerites ${ }^{8}$ or in the red coral skeleton ${ }^{15 c}$. This fact supports the idea that the degree of misorientation (or misalignment) is limited, possibly controlled by crystallographic constraints. The reason why misorientations would reach a maximum (for instance in the frame) and would not tend towards spherulitic structure is not yet understood. On another side, the answer to the question could be 'No' since misorientation limitations could be overruled by mesotwinning and branching. For 
instance, in the deeply rooted tubercles, large misorientations $\left(+/-50^{\circ}\right)$ can be reached step by step through structural branching (Fig. 12g). By a fractal game, a range of misorientations larger than $+/-22^{\circ}$ can be reached in tripod superstructures, allowing the crystallite $c$ axes to stay perpendicular to the sclerite growth front, even in the case of corrugated surfaces. Summarizing, from a practical point of view, it is not relevant to include a maximum value of crystallite misalignment in the definition of mesocrystal.

An interesting conclusion of the present work is that crystalline order persists between mesocrystalline domains over long distances (at $\mathrm{mm}$ scale). In this respect, the octocoral sclerites display a diversity of crystallographic singularities between two domains: (1) similarity of three independent row directions (the crystallites are similarly oriented), (2) similarity of two independent row directions in the same plane and apposition of this plane (mesotwinning), (3) similarity of two independent row directions without apposition of the plane (as in the frame and tubercle interface), or (4) similarity of a single row direction (as in Fig $5 \mathrm{e}$ from ${ }^{8 \mathrm{a}}$ in the red coral case). These crystallographic singularities indicate that a large spectrum of crystallographic configurations come into play to minimize crystallographic disorder in the hierarchical structure of octocoral sclerites.

Morphology and crystallography: To directly answer a question raised in the introduction, the strict rules that govern crystal morphologies do not necessarily apply to assemblages of crystallites. Curvatures difficult to achieve in perfect crystals can be formed with assemblages of small crystals. The size of crystalline units and the possibility of misorientations and mesotwinning between them are key properties of octocoral biominerals to achieve all kinds of shapes and form adaptative structures. In other words, biominerals take advantage of defects (grain boundaries, misorientations, mesotwinning) to build hierarchical structures that still 
preserve crystallographic order. This conclusion suggests that defects are required to achieve crystalline hierarchical order.

The study of Sinularia sclerites also demonstrates that a combination of trigonal inverse pyramid allows the formation of growth fronts with complex shapes. To our knowledge, this inverse pyramid superstructure has not been evidenced so far. It could play an important role in the transition from relatively simple structures with one or two tripods (e.g. Sinularia proto sclerites or red coral dumbbell shape sclerites), to more complex structures with tripods in various spatial configurations (e.g. large Sinularia sclerites).

The views exposed above complement those of Addadi et al. ${ }^{2}$ and Mann ${ }^{1}$. Concerning ACC, an amorphous phase as a precursor of sclerites has not yet been fully characterized, but its presence in the scleroblast is likely ${ }^{20 a}, 21$. However, considering the observed symmetrydependant morphology and the mesocrystalline hierarchical organization, ACC is not expected to play a major role in the final morphology of octocoral sclerites. The proposal that mesocrystalline structures can form almost any shape remains consistent with Mann's view that biomineralized structures may originate from the regulation of crystal growth and patterning by organic assemblies. As seen above, concave spaces $(\sim 20-30 \mu \mathrm{m}$ wide) generated by the alternation of frame concavities and tubercles (Fig. 4c) could be occupied by mineralizing tissues, cells or multi-cellular vacuoles at the sclerite surface. Therefore, transposing Mann's idea to a larger scale, surface morphology of the sclerite could in part result from the regulation of mesocrystalline growth and patterning by multi cellular mineralizing epithelium. The biomineral morphologies of octocoral skeleton and sclerites would thus arise in part from biological patterning ultimately programmed by genetic coding. In the end, the morphology of the Sinularia sclerites could be the consequence of both internal and external forces, among which crystallographic order and external molding play important roles. 


\section{Acknowledgments}

The title of this article refers to the inspiring work of S. Mann on 'The chemistry of form'. This work has been supported by the Centre National de la Recherche Scientifique (CNRS), by Institut National des Sciences de l'Univers (INSU) through grant INTERRVIE 2017, by the Agence National pour la Recherche (ANR) through ANR CoRo 2011-2015, by the Centre Interdisciplinaire de Nanosciences de Marseille (CINaM), and by the European Union COST action TD0903. This work has been partly carried out within the framework of the ICoME2 Labex (ANR-11-LABX-0053) and the A*MIDEX projects (ANR-11-IDEX-0001-02) cofunded by the French programme 'Investissements d'Avenir', managed by the ANR, the French National Research Agency. Observations were made on a FESEM financed by the European Fund for Regional Development (EFRD). We thank F. Bedu, I. Ozerov and D. Chaudanson for their assistance on the CINaM SEMs. EBSD analyses were carried out at the Institut National des Sciences de l'Univers facility at Geosciences Montpellier, France; we thank F. Barou for his help. EMP maps and analyses were obtained at Laboratoire Magmas et Volcans (Clermont-Ferrand France) with the help of J.L. Devidal. Reviews by two anonymous reveiwers as well as editorial handling by A.V. Mudring are gratefully acknowledged. This is contribution ANR CoRo ${ }^{\circ} 10$.

\section{Supporting information description}

SI\#1: Sclerites in the tissues of Sinularia polydactyla. SI\#2: BSE-SEM images of longitudinal sections of two coalesced large sclerites. SI\#3: EMP map of magnesium in a large Sinularia sclerite. SI\#4: Physical meaning of the variation of contrast in a sclerite under polarizing microscope. SI\#5: Stereographic projection of the sclerite frame from Fig. 9d. SI\#6: Stereographic projection of the tubercle from Fig. 12. 


\section{Author Information}

Phone: 33662922841. Fax: 33491418916. Email: vielzeuf@ cinam.univ-mrs.fr

\section{References}

1. Mann, S., The chemistry of form. Angewandte Chemie-International Edition 2000, 39, 3393-3406.

2. $\quad$ Addadi, L.; Raz, S.; Weiner, S., Taking advantage of disorder: Amorphous calcium carbonate and its roles in biomineralization. Advanced Materials 2003, 15, 959-970.

3. Weiner, S.; Sagi, I.; Addadi, L., Choosing the crystallization path less traveled. Science 2005, 309, 1027-1028.

4. $\quad$ (a) Wang, D. B.; Hamm, L. M.; Bodnar, R. J.; Dove, P. M., Raman spectroscopic characterization of the magnesium content in amorphous calcium carbonates. Journal of Raman Spectroscopy 2012, 43, 543-548; (b) Zolotoyabko, E.; Caspi, E. N.; Fieramosca, J. S.; Von Dreele, R. B.; Marin, F.; Mor, G.; Addadi, L.; Weiner, S.; Politi, Y., Differences between Bond Lengths in Biogenic and Geological Calcite. Crystal Growth \& Design 2010, 10, 1207-1214; (c) Pecher, J.; Guenoun, P.; Chevallard, C., Crystalline Calcium Carbonate Thin Film Formation through Interfacial Growth and Crystallization of Amorphous Microdomains. Crystal Growth \& Design 2009, 9, 1306-1311.

5. Towe, K. M., Sea urchins as crystallographers. Science 2006, 311, 1554-1555.

6. Schmidt, W. J., Die Bausteine des Tierkörpers in polarisiertem Lichte. . Verlag Friedrich Cohen: Bonn, 1924.

7. Bengtson, S., Atractosella, a Silurian Alcyonacean octocoral. J. Paleontol. 1981, 55, 281294.

8. (a) Floquet, N.; Vielzeuf, D., Mesoscale twinning and crystallographic registers in biominerals. American Mineralogist 2011, 96, 1228-1237; (b) Floquet, N.; Vielzeuf, D., Ordered Misorientations and Preferential Directions of Growth in Mesocrystalline Red Coral Sclerites. Crystal Growth \& Design 2012, 12, 4805-4820.

9. Perrin, J. Structure et squelettogenèse chez le genre Corallium. Aix-Marseille Université, 2014.

10. (a) Bayer, F. M., Octocorallia. Geological Society of America and University of Kansas Press: Lawrence, 1956; p F166-F231; (b) Goldberg, W. M.; Benayahu, Y., Spicule formation in the Gorgonian coral Pseudoplexaura-Flagellosa. 1: Demonstration of the intracellular and extracellular growth and the effect of ruthenium red during decalcification. Bulletin of Marine Science 1987, 40, 287-303.

11. Majoran, S., Structural investigations of Octocoral sclerites. Zoologica Scripta 1987, 16, 277-287.

12. Sethmann, I.; Helbig, U.; Worheide, G., Octocoral sclerite ultrastructures and experimental approach to underlying biomineralisation principles. CrystEngComm 2007, 9, 1262 1268.

13. Tentori, E.; van Ofwegen, L. P., Patterns of Distribution of Calcite Crystals in Soft Corals Sclerites. Journal of Morphology 2011, 272, 614-628. 
14. Perrin, J.; Vielzeuf, D.; Ricolleau, A.; Dallaporta, H.; Valton, S.; Floquet, N., Block-byblock and layer-by-layer growth modes in coral skeletons. American Mineralogist 2015, 100, 681-695.

15. (a) Griesshaber, E.; Schmahl, W. W.; Neuser, R.; Pettke, T.; Bluem, M.; Mutterlose, J.; Brand, U., Crystallographic texture and microstructure of terebratulide brachiopod shell calcite: An optimized materials design with hierarchical architecture. American Mineralogist 2007, 92, 722-734; (b) Dalbeck, P.; England, J.; Cusack, M.; Lee, M. R.; Fallick, A. E., Crystallography and chemistry of the calcium carbonate polymorph switch in M. edulis shells. European Journal of Mineralogy 2006, 18, 601-609; (c) Vielzeuf, D.; Floquet, N.; Chatain, D.; Bonneté, F.; Ferry, D.; Garrabou, J.; Stolper, E. M., Multilevel modular mesocrystalline organization in red coral. American Mineralogist 2010, 95, 242-248.

16. Massaro, F. R.; Pastero, L.; Rubbo, M.; Aquilano, D., Theoretical surface morphology of \{01-12\} acute Rhombohedron of calcite - A comparison with experiments and $\{10-14\}$ cleavage rhombohedron. Journal of Crystal Growth 2008, 310, 706-715.

17. (a) Downs, R. T.; Hazen, R. M., Chiral indices of crystalline surfaces as a measure enantioselective potential. Journal of Molecular Catalysis a-Chemical 2004, 216, 273-285; (b) Hazen, R. M., Mineral surfaces and the prebiotic selection and organization of biomolecules. American Mineralogist 2006, 91, 1715-1729.

18. Kingsley, R.; Watabe, N., Ultrastructural investigation of spicule formation in the gorgonian Leptogorgia virgulata (Lamarck) (Coelenterata: Gorgonacea). Cell and Tissue Research 1982, 223, 325-334.

19. Jeng, M. S.; Huang, H. D.; Dai, C. F.; Hsiao, Y. C.; Benayahu, Y., Sclerite calcification and reef-building in the fleshy octocoral genus Sinularia (Octocorallia: Alcyonacea). Coral Reefs 2011, 30, 925-933.

20. (a) Tixier-Durivault, A., Contribution à l'étude du métabolisme du calcium et du fer chez l'Alcyonium palmatum (Pallas). Annales de l'Institut Océanographique 1940, 20, 311-379; (b)

Dunkelberger, D. G.; Watabe, N., An ultrastructural study on spicule formation in the pennatulid colony Renilla reniformis. Tissue \& cell 1974, 6, 573-86; (c) Kingsley, R. J.; Watabe, N., Synthesis and transport of the organic matrix of the spicules in the gorgonian Leptogorgia virgulata (Lamarck) (Coelenterata, Gorgonacea) - An autoradiographic investigation. Cell and Tissue Research 1984, 235, 533-538.

21. Tixier-Durivault, A., Sous-classe des Octocoralliaires. In Traité de Zoologie. Anatomie, Systématique, Biologie, Grassé, P.-P., Ed. Masson: Paris, 1987; Vol. III, 3, pp 3-185.

22. Lowenstam, H. A.; Weiner, S., On biomineralization. Oxford University Press: Oxford, 1989.

23. Cölfen, H.; Mann, S., Higher-Order Organization by Mesoscale Self-Assembly and Transformation of Hybrid Nanostructures. Angewandte Chemie International Edition 2003, 42, 2350-2365.

24. (a) Cölfen, H.; Antonietti, M., Mesocrystals and Nonclassical Crystallization. In Mesocrystals and Nonclassical Crystallization, John Wiley \& Sons, Ltd: 2008; pp 1-6; (b) Cölfen, H.; Antonietti, M., Mesocrystals: Inorganic superstructures made by highly parallel crystallization and controlled alignment. Angewandte Chemie-International Edition 2005, 44, 5576-5591; (c) Song, R.-Q.; Cölfen, H., Mesocrystals-Ordered Nanoparticle Superstructures. Advanced Materials 2010, 22, 1301-1330; (d) Niederberger, M.; Cölfen, H., Oriented attachment and mesocrystals: Non-classical crystallization mechanisms based on nanoparticle assembly. Physical Chemistry Chemical Physics 2006, 8, 3271-3287. 
25. (a) Bailey, J. K.; Brinker, C. J.; Mecartney, M. L., Growth mechanisms of iron-oxide particles of differing morphologies from the forced hydrolysis of ferric-chloride solutions. Journal of Colloid and Interface Science 1993, 157, 1-13; (b) Privman, V.; Goia, D. V.; Park, J.; Matijevic, E., Mechanism of formation of monodispersed colloids by aggregation of nanosize precursors. Journal of Colloid and Interface Science 1999, 213, 36-45; (c) Collier, C. P.; Vossmeyer, T.; Heath, J. R., Nanocrystal superlattices. Annual Review of Physical Chemistry 1998, 49, 371-404.

26. Kim, Y. Y.; Schenk, A. S.; Ihli, J.; Kulak, A. N.; Hetherington, N. B. J.; Tang, C. C.; Schmahl, W. W.; Griesshaber, E.; Hyett, G.; Meldrum, F. C., A critical analysis of calcium carbonate mesocrystals. Nature Communications 2014, 5.

27. Bergstrom, L.; Sturm Nee Rosseeva, E. V.; Salazar-Alvarez, G.; Coelfen, H., Mesocrystals in Biominerals and Colloidal Arrays. Accounts of Chemical Research 2015, 48, 1391-1402.

28. Okumura, T.; Suzuki, M.; Nagasawa, H.; Kogure, T., Characteristics of biogenic calcite in the prismatic layer of a pearl oyster, Pinctada fucata. Micron 2010, 41, 821-826.

29. Cölfen, H., Precipitation of carbonates: recent progress in controlled production of complex shapes. Current Opinion in Colloid \& Interface Science 2003, 8, 23-31.

\section{For Table of Contents Use Only}

\section{The Crystallography of complex forms: the case of octocoral sclerites}

Daniel Vielzeuf, Nicole Floquet, Jonathan Perrin, Eric Tambutté, and Angèle Ricolleau

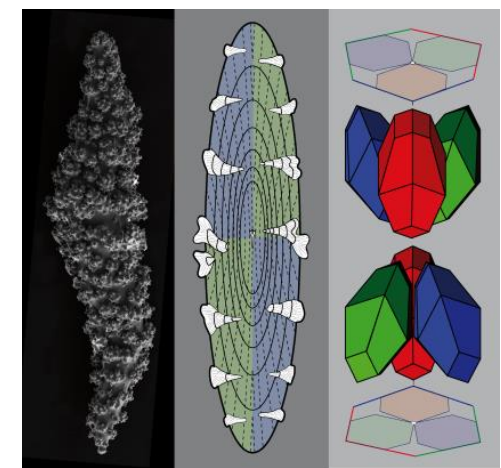

Many biominerals display hierarchical organization but little is known about the transition from simple to complex crystallographic structures. With the example of octocoral sclerites, we show that some biominerals combine crystallographic order and disorder to achieve all kinds of shapes. Sclerite morphologies are the product of internal and external forces, among which crystallographic branching and external molding play important roles. 